\title{
Application of periodic boundary conditions on multiple part finite element meshes for the meso-scale homogenization of textile fabric composites
}

\author{
S. Jacques $^{1}{ }^{*}$, I. De Baere ${ }^{1}$ and W. Van Paepegem ${ }^{1}$
}

${ }^{1}$ Ghent University, Dept. of Materials Science and Engineering, Technologiepark-Zwijnaarde 903, 9052 Zwijnaarde, Belgium

*corresponding author: Stefan.Jacques@ UGent.be; T +3293310435

\begin{abstract}
Textile fabric reinforced composites are increasingly used in structural applications in the aerospace, automotive and recreational industry. Since experimental testing is labour intensive and time consuming, numerical analysis using Representative Unit Cell (RUC) and Finite Element (FE) analyses for obtaining the elastic material constants have proven to be suitable. One of the drawbacks of the existing techniques is that one is obliged to have identical meshes on opposite faces for applying periodic boundary conditions (PBC), or that multiple part finite element meshes are not allowed. The new ORAS software discussed in this paper allows non-identical meshes at opposite faces and multiple part meshes. If a search is done in the ISI web of knowledge, no papers can be found of the meso-scale finite element modelling with periodic boundary conditions of spread tow fabric composites. With the existing techniques available on the market it is not possible, and therefore the method presented in this paper gives a solution. For the numerical meso-scale FE analysis in combination with macro homogenization for obtaining the macro elastic constants, a thermoplastic carbon-PPS (PolyPhenylene Sulfide) 5-harness satin weave composite $\left(\right.$ CETEX $\left.^{\circledR}\right)$ was used as an example. The results of the meso-scale FE analysis of the RUC using PBC with macro homogenization obtained with the new technique are in good agreement with those obtained using conventional techniques and experimental data.
\end{abstract}

Keywords: A. Textile composites; B. Mechanical properties; C. Finite element analysis (FEA); C. Multiscale modelling; Periodic Boundary Conditions 


\section{Introduction}

The mechanical behaviour of textile fabric composites is complex because it is a multi-scale problem. The macroscopic behaviour is very dependent on the interactions of the yarns and the matrix at meso-scale (scale of the textile unit cell). Similarly, the behaviour of the unit cell at meso-scale is dependent on the interactions of fibres and fibres and matrix at the interface at micro-scale level. Over the years numerous approaches were developed in order to predict the mechanical behaviour of textile reinforced composites. Depending on the complexity of the architecture of the reinforcement, more or less complicated methods were introduced starting from simple analytical equivalent laminate models to complex "cells" based models representing the $3 \mathrm{D}$ geometry of the textile architecture [1,2,3]. Another approach with approximate representation of the reinforcing geometry for obtaining the homogenized elastic properties of the composite RUC uses the averaged properties of differently oriented yarns in the architecture based on the transformation of the stiffness tensor with the reference coordinate system, with the inclusion-based model as a generalisation of this approach $[1,3,4]$. In order to capture the complex stress-strain fields throughout the RUC, many researches explored the possibility of using the FE calculations. Work from Kabelka (1984) [5], Woo and Whitcomb (1992) [6], Sankar and Marrey (1997) [7] presented solutions for 2D analyses of plain weave composites using the assumption of plain-strain state, but these models are not suitable for correctly modelling textile composites [3]. Yoshino and Ohtsuka (1982) [8], Whitcomb (1989) [9], Dasgupta et al. (1994) [10], Naik and Ganesh (1992) [11], Paumelle et al. (1991) [12], Blacketter et al. (1993) [13], Glaesgen et al. (1996) [14], MCilhagger and Hill et al. (1995) [15], Lomov et al. (2005) [16], Verpoest and Lomov (2005) [17] and Kurashiki et al. (2005) [18] developed 3D models in combination with homogenization theories viz. kinematic and periodic boundary conditions for the prediction of the macro homogenized elastic properties of textile reinforced RUC. One of the big issues when using any of the 3D models of the reinforcement is correctly defining and modelling the reinforcement architecture since all models use mathematically simplified representations of the cross sections of the yarns (circular, elliptical, lenticular or polygonal) [3]. This leads typically to an 
underestimation of the fibre volume fractions. Another reason is that for existing $\mathrm{PBC}$ techniques either identical meshes at opposite faces are needed with a single part mesh $[1,19]$, either non-identical meshes at opposite faces can be handled but a unique part mesh is needed or unique material is needed. This uniqueness of the parts mesh or material is the drawback of the methods defined in [20,21]. For example, if one tries to find papers concerning the unit cell modelling of spread tow composites in the ISI web of knowledge database, one will see that no reference can be found yet. A spread tow typically has a very high width to height ratio leading to very small matrix pockets at the cross over points of the spread tow fabric composite. The commercial finite element software packages creating meso-scale FE models of these spread tow fabric composites unit cell will lead to an assembly of multiple mesh parts (yarns and matrix). Such a complex unit cell with different mesh parts, often composed of different element types (tetrahedrons and hexahedrons), will consist of non-identical meshes at opposite faces of the unit cell, with interactions at the interfaces between the different parts. No available technique can handle these difficulties of such a unit cell in a meso-scale FE simulation with periodic boundary conditions. Therefore this paper will present a method allowing the FE meso-scale calculation using PBC with non-identical meshes at opposite faces and allowing multiple parts meshes and multiple materials. The new technique is benchmarked with state of the art techniques and experimental results [22] on a 5 harness satin weave composite and at the end the potential of the presented method is shown on a spread tow fabric composite model.

The following six main sections will be addressed: i) material properties extraction using a micro-CT scan; ii) creation of the geometrical model of the RUC with commercial CAD software; iii) mesh generation of the CAD model in a commercial FE software; iv) applying local orthotropic material properties respecting the orientation of the yarns and the volume fractions (fibre, matrix) using developed software (C\# code); v) PBC creation using developed software (C\# code); vi) macro homogenization of the composite stiffness using the FE results. 
Advantages of this new approach are: i) no restrictions for yarn shapes (cross section/ undulation) and matrix (voids); ii) use a micro-CT scan as input for CAD generation; iii) all different meshes (tetrahedral, hexahedral) can be handled using FE software; iv) interface layers (cohesive elements) can be implemented easily for the modelling of damage in between the yarns/matrix, yarns/yarns or in the matrix itself.

All functionalities have been grouped in the in-house developed ORAS software (Object oriented, RVE, Assembly, Software).

\section{Unit cell modelling}

\subsection{Material Properties}

For this research work, the example material used is a thermoplastic 5-harness satin weave composite (CETEX $\left.{ }^{\circledR}\right)$ with T300JB carbon fibre as reinforcement and PPS (PolyPhenylene Sulfide) as matrix. The fabric geometrical parameters needed for the construction of the RUC geometry such as yarn spacing, yarn width and thickness of the yarns can be found in Table 1, and were extracted from a micro-CT analysis [22] (Figure 1), together with the parameters of the constituents (carbon fibre and PPS matrix). The material properties of the constituents of the textile composite, the T300JB carbon fibre $(\mathrm{E} 11=231 \mathrm{GPa}$; E22 $=28 \mathrm{GPa}$; G12 $=24$ $\mathrm{GPa} ; \mathrm{G} 23=10.7 \mathrm{GPa} ; v 12=0.26 ; v 23=0.3)$ and the PPS resin $(\mathrm{E}=3.8 \mathrm{GPa} ; \mathrm{G}=1.38 \mathrm{GPa}$; $v=0.37$ ), are used to calculate the impregnated carbon-PPS unidirectional composite material properties $(\mathrm{E} 11=162.60 \mathrm{GPa} ; \mathrm{E} 22=13.70 \mathrm{GPa} ; \mathrm{G} 12=6.50 \mathrm{GPa} ; \mathrm{G} 23=5.07 \mathrm{GPa} ; v 12=$ 0.29 ; $v 23=0.35)$ using the analytical Chamis micro-mechanical homogenization formulas [23] (Equations ((2-1)- (2-6)) with an intra-yarn fibre volume fraction $\mathrm{K}_{\mathrm{f}}=0.7$.

Longitudinal modulus: $\quad E_{11}=K_{f} E_{f 11}+K_{m} E_{m}$

Transverse modulus : $\quad E_{22}=\frac{E_{m}}{1-\sqrt{K_{f}}\left(1-\frac{E_{m}}{E_{f 22}}\right)}=E_{l 33}$

Shear modulus :

$$
G_{12}=\frac{G_{m}}{1-\sqrt{K_{f}}\left(1-\frac{G_{m}}{G_{f 12}}\right)}=G_{l 13}
$$


Shear modulus :

$$
G_{23}=\frac{G_{m}}{1-\sqrt{K_{f}}\left(1-\frac{G_{m}}{G_{f 13}}\right)}
$$

Poisson's ratio:

$$
v_{12}=K_{f} v_{f 12}+K_{m} v_{m}=v_{l 13}
$$

Poisson's ratio:

$$
v_{23}=\frac{E_{l 22}}{2 G_{l 23}}-1
$$

where coordinate 1 represents the fibre direction

\subsection{Geometrical model}

Specialized geometric model pre-processors for building models of the internal structure of textile reinforcements can be found nowadays, with the most well-known being WiseTex and TexGen [17,24]. Numerous geometries of textile architectures (woven, braided, NCF, knitted...) can be created using those software products, but in most cases only ideal yarn shapes and architectures can be developed without taking into account real deformation and positioning of the yarns in a real composite structure. For many research purposes, one is interested in the impact of the deviation of the geometry compared to the ideal models [25]. The creation of the geometry using commercial CAD software allows having the freedom of creating any yarn shape in longitudinal and transverse directions and it allows the integration of defects in the matrix e.g. voids. The possibility to import '.stl'-files in the CAD software, opens possibilities to use a micro-CT analysis to build up the geometrical model of a real composite. A '.stl' or standard tessellation language file is an ASCII or binary file in which the 3D shape of a geometry is described by a raw unstructured triangulation of the surface. The surface of the geometry is discretised in triangles. Two models ('Model A' with yarn height $=0.155 \mathrm{~mm}$ and 'Model B' with yarn height $=0.156 \mathrm{~mm}$ ) of the RUC (Figure 2) are made in Catia V5 using the parameters of yarns and matrix as given in section 2.1, staying within the limits given in Table 1 . The choice of these values was made in order to be able to compare the method developed in this paper to the state of the art technique, according to the fibre volume fractions after meshing. In Table 3 one can see that the obtained fibre volume fractions for the 3 models (WiseTex model, Model A and Model B) are close to each other. Additionally the difference between the yarn heights of 'Model A' and 'Model B' was 
implemented in order to study the influence of a small increase of the thickness of the yarns on the global material behaviour. A yarn volume is obtained by two sequential steps. An elliptical shape was chosen as cross section (= sweep profile) after analysing the micro-CT scan (Figure 1 (c)), followed by a sweep operation along the heart line (= sweep path) of a yarn (Figure 3(b)). The yarn cross sectional shape, the yarn width, the yarn thickness and the inter yarn spacing will induce the shape of the heart line. Line segments perpendicular to these cross sections (Figure 3(a)) are created with the start point on the cross sections and with the end point at distance $h=\frac{t_{\text {yarn }}}{2}+t_{\text {Matrix }}$, where $t_{\text {Matrix }}$ represents the thickness of the matrix layer in between the yarns (Figure 3(a)(c)). At the cross over points, the spline point at the centre is defined by the midpoint between point 1 and point 2 of the cross section extremes in Figure 3(a). Since WiseTex/MeshTex does not allow changes in cross sections along the heart line and variations in the alignments of the yarns (Figure 1), it was chosen to keep a similar idealized model in this paper as can be obtained with WiseTex/MeshTex. The purpose is to validate the proposed method with an existing and widely used technique. The geometrical models created, were imported in a commercially available FE software and partitions were made at all boundary faces but top and bottom face (in direction 3) (see section 2.5.3.1)

\subsection{Mesh}

The geometrical models created in the previous step are meshed with the pre-processor of a commercial FE software (Abaqus ${ }^{\mathrm{TM}}$ ). The yarns were meshed using an advancing front sweep mesh. A 3D 8-node (three translational degrees of freedom per node) linear structural solid element is used. The matrix has been meshed with 3D 4-node (three translational degrees of freedom per node) linear tetrahedral elements in order to catch the curvatures of the model (Figure 4 (a)(b)(c)(d)). Four mesh models are built 'A-M1', ' B-M2', 'B-M3' and 'B-M4' out of the geometrical models 'Model A' and 'Model B', with the mesh sizes for 'AM1' and ' B-M2' (Figure 5) similar to the benchmarked model mesh obtained using the MeshTex software [16,18,22]. Since mesh convergence could have an impact on the fibre 
volume fraction, on the FE results and thus on the homogenized elastic properties for the macro scale composite, model 'B-M3' with the same mesh for the matrix, but with an increased number of elements for the yarn's mesh (Figure 5 and Figure 4(a)(c)(d)) is created in order to capture the influence of the mesh size of the yarns. Finally in the mesh model 'BM4' the same yarn mesh as for model 'B-M3' is used with a refined matrix mesh (Figure 5 and Figure 4(b)(c)).

2.4. Application of the local orthotropic material properties respecting the orientation of the yarns

Each contour of a yarn is swept along a heart line in order to create a model of the yarns in section 2.2 using CAD software. Therefore one will use this heart line to create the different local transverse isotropic orientations of the carbon fibre yarns. The heart line is meshed in a user chosen FE software and the elements and nodes (wires) are extracted and written to a text file using a python script. A procedure written in a multithreaded C\# code (Figure 6(a)) allows the user to choose the input files (nodes and elements) of the yarns mesh and of the heart line mesh. The first direction (along the fibre) corresponds to the direction defined by the 2 nodes (A and B) of a mesh element $(h)$ of the heart line (Figure 6(a)). In the centre point of each element a plane normal to the first direction is created on which the fibre direction 2 and 3 are created in order to create an orthogonal axis system. The centroid $\left(C^{n}\right)$ of each element $n$ of the yarn mesh is projected on the centre points $\left(C^{h}\right)$ of all heart line mesh elements. If the distance $\left|C^{n} C^{h}\right|$ calculated between both centroids is minimal using equation (2-7), the orientation of that element is defined by the directions created at the corresponding mesh element of the heart line.

$$
\operatorname{Min}\left\{d\left(\left|C_{n} C_{h}\right|\right) \mid d=\sqrt{\left(x_{1}-x_{2}\right)^{2}+\left(y_{1}-y_{2}\right)^{2}+\left(z_{1}-z_{2}\right)^{2}}\right\}
$$

The algorithm for the material orientation definition is applicable on all 3D meshes (tetrahedral, hexahedral, linear and quadratic). Resulting sets, orientations and sections are written to a text file with the user chosen material. 


\subsection{Boundary conditions}

\subsubsection{Periodic boundary conditions}

The basic idea of using periodic boundary conditions is to assume that a part on macro level consists of a number of repeated RUC's in which each basic mechanical element, the RUC, determines the global constitutive law of the material on macro level (Figure 2) [1,26,27]. This implies that continuity of the displacements at neighbouring faces of the RUC's must be fulfilled and thus any displacement on one side of the RUC must be the same on the opposite side plus or minus some constant $[1,27,28]$. Not taking into account the rigid displacements and rotations of the RUC, the displacement field for a periodic structure is related to the strain field by the expression:

$$
u(\bar{x})=\overline{\bar{\varepsilon}} \bar{x}+\tilde{u}(\bar{x})
$$

where $\overline{\bar{\varepsilon}}$ in the first term represents the macroscopic strain tensor and $\bar{x}$ the position vector of a material point in the RUC. The second term represents a volume periodic term with zero average value with $\tilde{u}$ being the local displacement field in the RUC. A second condition that has to be met is the anti-periodicity of the traction distributions at the opposite boundaries of the RUC $(\partial \mathrm{V})$ :

$$
\bar{t}=\overline{\bar{\sigma}} \bar{n}
$$

Considering the RUC in Figure 2, when substituting the macroscopic displacement gradients of the unit cell [22] into the periodic equations, one obtains the nine periodic conditions (Table 2) using the axis system as given in Figure 2. The state of the art requires exactly identical meshes on opposite faces of the RUC [1,19]. The current approach offers a new solution in order to allow non-identical meshes and multiple part meshes to be used in PBC. Section 2.5.2 explains this procedure.

\subsubsection{New ORAS software for the implementation of PBC with non-identical meshes at opposite faces in a RUC}

If one considers the architecture of a RUC of a real textile fabric composite unit cell, the yarns in the matrix can have complex shapes with variations in thickness and undulations. Or the 
amount of matrix in between the yarns can be very low with complex pockets in the cross over points of the yarns. When importing a CAD model of such a RUC into commercially available FE software, the model will consist of multiple parts in an assembly. Even if the cross sections of the yarns and thus the matrix at the opposite faces will be identical, the mesh generated of this assembly will automatically generate parts meshes with different amounts of nodes at the opposite faces due to the complexity of the matrix mesh. Often, for very complex models, the matrix will exist of tetrahedral meshes with non-identical amounts of nodes at opposite faces. In this section a solution will be given for allowing such meshes in PBC definitions making use of the following steps:

1. Creation of a grid comprising all mesh nodes of the domain $\partial \mathrm{V}$ of the RUC

2. Definition of reference points for each grid section

3. Constraint definitions at the interfaces

Steps 1 and 2 were implemented in the C\# code ORAS in order to obtain the sections of the input files concerning the periodic boundary conditions automatically with minimal user inputs.

\subsubsection{Creation of a grid of $\partial \mathrm{V}$}

Considering the volume $\mathrm{V}$ of a mesh, with two opposite boundary domains $\partial \Omega_{1}$ and $\partial \Omega_{2}$ (the respective faces $A B C D$ and $A^{*} B^{*} C^{*} D^{*}$ (Figure $\left.6(b)\right)$ ), a grid can be made using a user defined grid size for the $\mathrm{x}$, respectively the $\mathrm{y}$ direction:

$$
\begin{aligned}
\Delta X_{i}=X_{i}-X_{i-1} ; & \Delta Y_{j}=Y_{j}-Y_{j-1} ; \\
\Delta X_{i+1}=X_{i+1}-X_{i} ; & \Delta Y_{j+1}=Y_{j+1}-Y_{j} ;
\end{aligned} \quad \text { with } \mathrm{r}_{\mathrm{i}}=\frac{\Delta \mathrm{x}_{\mathrm{i}+1}}{\Delta \mathrm{x}_{\mathrm{i}}} ; \mathrm{r}_{\mathrm{j}}=\frac{\Delta \mathrm{Y}_{\mathrm{j}+1}}{\Delta \mathrm{Y}_{\mathrm{j}}}
$$

Using a uniform PBC grid size, $r_{i}=r_{j}=1$, all mesh nodes of $\partial \mathrm{V}$ can be distributed into $n$ cells. Since the same grid is used for opposite domains $\partial \Omega_{1}$ and $\partial \Omega_{2}$, the corresponding cells at opposite faces will contain associated mesh nodes. The nodes of the corresponding cells at opposite faces will be given $\mathrm{PBC}$ with the technique explained in section 2.5.2.2.

In order to investigate the influence of the PBC grid size, three different uniform PBC grid sizes are used in the FE calculations, with $\Delta X_{i}=\Delta Y_{i}$, given in Figure 5. 


\subsubsection{Definition of the reference points}

In current PBC techniques with identical meshes at opposite faces, each node of one face is linked to the corresponding node at the opposite face using the PBC constraint as defined in section 2.5.1. If the number of nodes in cell $k$ of domain $\partial \Omega_{1}$ (Figure 6(c)) of the new PBC technique differs from the number of nodes in the corresponding cell $k^{*}$ of domain $\partial \Omega_{2}$, the associated nodes cannot directly be linked to each other using the PBC constraint due to the overconstraint of the nodes. In the FE software products it is not allowed to have more than one PBC equation definition at one node of the FE mesh. To avoid this problem, cell $k$ containing the mesh nodes $p_{i}$ with $i=1,2,3$ will be linked to a reference node $p$. The spatial coordinates of the reference nodes are obtained by the Laplacian average:

$$
p=\frac{\sum_{i=1}^{n} p_{i}}{n}
$$

If the uniform grid size chosen is very small, with a $\Delta X_{i}$ smaller than the smallest distance between 2 nodes of the same face, one could obtain a grid cell $k$ in domain $\partial \Omega_{1}$ containing mesh nodes and an empty associated cell $k^{*}$ of domain $\partial \Omega_{2}$. From the floating node $p_{n}\left(x_{1}, y_{1}\right)$ of cell $k$ of $\partial \Omega_{1}$ (node without associated nodes in the associated cell $k^{*}$ of $\partial \Omega_{2}$ ) (Figure 6 (c)) a circular area $\Gamma$ with a user chosen radius $R$ (with $\mathrm{R}>2 \Delta X_{i}$ ) is created. The size of $R$ has to be large enough compared to the FE mesh element size in order to find nodes inside the circular area $\Gamma$. For all nodes $p \in \Gamma$, one calculates the following objective function (Equation (2-11)) in order to obtain the mesh node $p_{y} \neq p_{n}$ the closest to $p_{n}$ :

$$
\operatorname{Min}\left\{\mathrm{D}\left(\mathrm{p}\left(\mathrm{x}_{\mathrm{i}}, \mathrm{y}_{\mathrm{j}}\right), \mathrm{p}_{\mathrm{y}}\left(\left(\mathrm{x}_{1}, \mathrm{y}_{1}\right)\right)\right) \text { with } \mathrm{p} \in \Gamma \mid \mathrm{D}=\sqrt{\left(\mathrm{x}_{1}-\mathrm{x}_{\mathrm{i}}\right)^{2}+\left(\mathrm{y}_{1}-\mathrm{y}_{\mathrm{i}}\right)^{2}}\right\}
$$

The larger $R$, the more nodes will be implemented in the search algorithm and equation (2-11), the higher the computational time for obtaining the closest point $p_{y}$. The node $p_{y}$ is associated with cell $j$ (Figure 6(c)) and node $p_{n}$ is implemented in the same cell. A new reference point using the Laplacian average method (Equation (2-10)) including pn in its calculation is obtained. All reference points are linked to the nodes of the corresponding cell using a tie constraint and are then linked to the equivalent reference points of the cells of the opposite domain using the PBC equation (section 2.5.1). The grid size used will have an 
influence on the results, since the bigger the grid, the more nodes each cell will contain and the higher the leverage on the nodes associated to a reference point (Figure 7(i)). This technique can be implemented for the different parts meshes of an assembly, and moreover with meshes where nodes are not shared at the interfaces (viz. the interface between matrix and yarns) the technique can be applied to each individual part mesh.

\subsubsection{Constraints of the interface surfaces}

Since the new technique allows the configuration with multiple parts in an assembly, constraints need to be defined at the interfaces between those parts like: tie constraints, contact definitions, cohesive elements... In the case of a meso-scale FE model, a tie constraint will mostly be chosen as interaction between yarns and matrix parts. This leads to overconstraining the nodes of domain $\partial \mathrm{V}$ at the interface between matrix and yarns since a node at the interface will be implemented in a tie constraint, a Multiple Point Constraint (MPC) constraint and periodic boundary conditions. An error will be generated in the FE software (Abaqus ${ }^{\mathrm{TM}}$ ) since the software will not know which of both constraints has priority, and therefore the displacement of the overconstrained node cannot be calculated. Two methods can be used in order to avoid the overconstraints of the nodes viz. section 2.5.3.1 and section 2.5.3.2.

\subsubsection{Partitioning the parts}

A partition near the boundary faces is made using a datum plane with a small offset $(0.010$ $\mathrm{mm}$ ) starting from the boundary face creating a layer of very small elements at the boundaries (Figure 8 (a)). The nodes of the elements at the interface of the multiple parts (matrix and yarns) are linked together with a tie constraint (Figure 8 (a-1)) except for the nodes laying on the domain $\partial \mathrm{V}$ (Figure 8(a-2)). The nodes on the boundary (domain $\partial \mathrm{V}$ ) will be linked with $\mathrm{PBC}$ equations to the nodes at the opposite face of the domain $\partial \mathrm{V}$. This procedure has no consequence on the results (see section 3) since the displacements generated by the PBC acting on the nodes of domain $\partial \mathrm{V}$ are transferred through the elements, with respect of the 
stiffness of the elements, to the rest of the model where tie constraints are acting between the multiple parts.

\subsubsection{Erasing the overconstrained nodes in the $\mathrm{PBC}$ equations and keeping the tie constraints}

For the meshes where partitioning is very complex and time consuming, the ORAS software offers the possibility to automatically erase the nodes, of the interface between yarns and matrix laying on the boundary domain $\partial \mathrm{V}$, from the $\mathrm{PBC}$ equation definitions (white and red dots in Figure 8(b)). The surrounding nodes of the domain $\partial \mathrm{V}$ (Figure 8 (b)), not laying at the interface between the parts, are still used in the PBC equations and will drive the displacements of the released nodes through the tie constraints. There is no influence on the results, as long as the mesh size is very fine at these interfaces of the multiple parts.

Both methods (section 2.5.3.1 and 2.5.3.2) allow the integration of layers, viz. cohesive zone models, at the interface of the multiple parts for the analysis of damage in the composite structure. Only the first method (section 2.5.3.1) was used for the meso-scale FE calculations of the validations discussed in section 3 .

\subsection{Macro-homogenization}

For the design and finite element calculation of composite parts of a structure at macro scale level, one needs to use homogenized mechanical properties. If one would build all models at meso-scale level, the calculation times needed for obtaining finite element responses for the behaviour of the composite components, would exceed the design time and the experimental testing time. Therefore at macro scale level, composite materials are considered as homogeneous. The relation between homogenized macro strains $\left(\varepsilon_{k l}^{H}\right)$ and macro stresses $\left(\sigma_{i j}^{H}\right)$ is given by: $\sigma_{i j}^{H}=C_{i j k l}^{H} \varepsilon_{k l}^{H}$ where $C_{i j k l}^{H}$ denotes the elasticity tensor at macro scale.

Following volume averaging technique:

$$
\langle\sigma\rangle=\frac{1}{V} \sum_{p=1}^{n} \sigma^{\mathrm{p}} V^{p}
$$


describes the volume averaging of the stresses over all integration points of all elements, where $V^{p}$ is the volume equivalent of the integration point. If the volume of the elements would be taken into account during the volume averaging of the stresses, the influence of a very big or very badly shaped element would be too big compared to other regular elements. In order to reduce the influence of these lower mesh quality elements on the stress results, the stresses are averaged by using the integration point volumes. To determine $C_{i j k l}^{H}$ starting from the FE model of the RUC using the periodic boundary conditions as described in section 2.5, six boundary value models $\varepsilon_{k l}(k, l=1 \ldots 3$ and $k \leq l)$ have to be solved. Out of the FE results of the six meso-scale models one calculates the homogenized stiffness

$$
C_{i j k l}^{H}=\frac{\left\langle\sigma_{i j}\right\rangle}{\varepsilon_{k l}} \delta_{i j} \delta_{k l}+\frac{\left\langle\sigma_{i j}\right\rangle}{2 \varepsilon_{k l}} \delta_{i k} \delta_{j l}\left|\delta_{i j}-1\right|
$$

After obtaining the compliance matrix $S_{i j k l}$ with a LU-decomposition [29], one calculates the elastic constants and Poisson ratio's.

\section{Validation}

In this section the following will be shown:

i) validation with experimental data

ii) validation of the new approach for the implementation of PBC with non-identical meshes at opposite faces using a WiseTex/ MeshTex model by comparison with the existing PBC implementation technique

iii) validation of the new technique for the construction of a FE model comprising multiple parts meshes using PBC constraints by comparison with the model obtained with WiseTex/ MeshTex

iv) comparison of the local strain fields of the WiseTex / MeshTex model and the model using the new ORAS software with experimentally measured strain profiles

v) implementation of periodic boundary conditions on a complex geometry, a spread tow reinforced composite, using the new technique 
3.1. Validation with experimental data

\subsubsection{Test setup and test samples}

In order to obtain the in-plane elastic properties of the satin weave composite specimens experimentally, tensile tests were made according to ASTM D3039 standard with measurement of the local strain with longitudinally and transversely applied strain gauges (Figure 9 (a)) using a servo-hydraulic Instron machine (8801, test speed $=2 \mathrm{~mm} / \mathrm{min}$ ) with a FastTrack 8800 digital controller and a load cell of $\pm 100 \mathrm{kN}$ [30]. The loads, displacements and strains, given by the FastTrack controller, as well as the extra signals from strain gauges were sampled on the same time basis and registered using National Instruments DAQ.

\subsubsection{Experimental results}

The results for the longitudinal and transverse modulus can be found in Figure 10(a) respectively (b) whereas the graph for the in-plane shear modulus and Poisson's ratio can be found in Figure 11(a) and (b). The summary of the obtained results are written in the last column of Table 4. It should be noted that catching all the anisotropic mechanical properties of a textile composite is a very labour intensive task and almost impossible to determine with the conventional experimental setups.

3.2. Validation of the ORAS software for the implementation of PBC by comparison with the existing $\mathrm{PBC}$ implementation technique (WiseTex/MeshTex) on the same mesh.

In order to validate the proposed PBC implementation, the new approach will first be benchmarked with the state of the art implementation. The model used for this validation is a 5H-satin weave unit cell created with WiseTex with the same RUC sizes as given in section 2.2 , but with an overall yarn thickness of $0.1545 \mathrm{~mm}$. The original yarn thickness of the WiseTex geometrical model is $0.159 \mathrm{~mm}$ and is artificially reduced by MeshTex in order to be able to create a mesh for the matrix. Only in the cross over points, the original $0.159 \mathrm{~mm}$ thickness for the yarns remains (Figure 4(e)) and thus altering the yarn cross section shape 
(Figure 4(f)). After meshing using MeshTex, the FE model consists of a single mesh part containing 53200 elements (37800 elements for the matrix) (Figure 4(e)). After transferring the models to a FE software, 8 node brick elements are assigned (C3D8) for the numerical simulation. The assignment of the transversely isotropic material properties to the yarns using the Chamis homogenized elastic properties of the carbon-PPS UD and isotropic material properties to the resin is made. For both the State of the Art (SotA) PBC implementation and the new approach using a coarse uniform PBC grid size G2 of 0.040mm (Figure 5), both applied on the same MeshTex FE mesh, the numerical results are compared and moreover a comparison with the experimental results (section 3.1) [22] is accomplished. The overall fibre volume fraction of the meshed unit cell calculated with the volume equivalent in all integration points (matrix and yarns) is equal to $47.2 \%$. The very small difference in homogenized elastic properties between the two approaches (column 1 'PBC ORAS' and column 2 'PBC SotA' of Table 4) is due to the coarse PBC grid and can be avoided if the PBC grid size $<$ smallest element edge in the model. In this case, since the smallest edge is approximately $9.10^{-5} \mathrm{~mm}$, the grid would be very dense and the calculation time for the implementation of the $\mathrm{PBC}$ would be very high for a negligible effective gain regarding the results in Table 4. The influence of the PBC grid size can be found in section 3.3.

3.3. Validation of the new ORAS software for the construction of a FE model comprising multiple parts meshes using PBC constraints by comparison with the model obtained using WiseTex/ MeshTex.

The nomenclature of the FE models used in this section with the ID's can be found in Figure 5, where 'A-M1-G2' stands for the FE mesh model 'A-M1' with a PBC grid size of 'G2'. The difference in yarn thickness of 'model A' and 'model B' described in section 2.2 and the MeshTex model can be explained by the fact that the original yarn thickness of the WiseTex geometrical model is $0.159 \mathrm{~mm}$ and is artificially reduced by MeshTex $(0.1545 \mathrm{~mm})$ in order to be able to create a mesh for the matrix. In order to be able to validate the new methodology, thicknesses of the yarns as given in section 2.2 were taken for 'model A' and 'model B' 
which correspond to equivalent fibre volume fractions as the one obtained using WiseTex/MeshTex (Table 3).

The FE results of the models defined in Figure 5, including the material properties for matrix and yarns and boundary conditions implemented using the new approach, are compared with the results obtained with the models defined in section 3.2 and the experimental results (section 3.1). Models 'A-M1-G2' and model 'B-M2-G2' are compared in order to see the impact of a small difference in the yarn thicknesses on the global macro-scale homogenized elastic constants. The difference in the results for model 'B-M2-G2', model 'B-M3-G2' and model 'B-M4-G2' shows the influence of the mesh size on the results, whereas the difference in results due to the PBC grid size can be noticed by comparing models 'B-M3-G1', 'B-M3G2' and 'B-M3-G3'.

The overall fibre volume fraction of the meshed unit cell (new approach) in a commercial FE pre-processor calculated with the volume equivalent in all integration points can be found in Table 3. The slightly higher fibre volume fraction for the new models can be explained by the differences in volumes of the yarns and matrix due to not sharing the nodes at the interface between the matrix and yarns in the models 'A-M1', 'B-M2', 'B-M3' and 'B-M4', whereas there are common nodes at the interface for the 'WiseTex/MeshTex' model in column 2. Not sharing the nodes at the interface creates 'empty spaces' or small penetrations depending on the density of the meshes at the interface, which explains the difference between the analytically calculated total volume for the RUC $(7.4 \mathrm{~mm} \times 7.4 \mathrm{~mm} \times 0.319 \mathrm{~mm}=17.46844$ $\mathrm{mm}^{3}$ ) and those obtained with assembly meshed models 'A-M1', 'B-M2', 'B-M3' and 'BM4'. Since the mesh of model 'B-M3' is finer concerning the yarns, with the same mesh for the matrix, than the one used for model 'B-M2', the volume obtained will be higher for the yarns. Model 'B-M4' with a very fine matrix mesh $(1,078,427$ elements) and same yarn meshes as model 'B-M3' approaches almost the analytically calculated volume. An infinitely small mesh would accurately correspond to the curve defined in the CAD model, but will lead to infinite calculation times. One can notice in Table 4 that the impact will be small for a mesh refinement of the FE model 'B-M2-G2' to model 'B-M3-G2' to model 'B-M4-G2', but 
could have an effect on the local strain contours. By intuition one would think that a coarser mesh provides higher stiffness due to the reduced deformation modes. But refinement of the mesh leads to an increase in the fibre volume fractions (Figure 12) and thus in stiffer results. A good agreement between model 'B-M4-G2', the experimental results and the state of the art WiseTex /MeshTex model can be found (Table 4). The higher $\mathrm{E}_{11}$ of the WiseTex/MeshTex model (+0.3 GPa) in column 3, knowing that the overall yarn thickness is lower $(0.1545 \mathrm{~mm})$ compared to $0.156 \mathrm{~mm}$ for the CAD model ' $\mathrm{B}$ ' and thus a lower fibre volume fraction, can be explained as follows:

- As explained in section 3.2, the mesh used in WiseTex/MeshTex has an overall yarn thickness of $0.1545 \mathrm{~mm}$ but in the cross over points of the yarns, these yarns have a thickness of $0.159 \mathrm{~mm}$ (Figure 4(e)(f)). The influence due to the decrease of the yarn thickness is not negligible as can be noticed by the increase of the stiffness $\mathrm{E}_{11}$ by 0.36 GPa between model 'A-M1-G2' and 'B-M2-G2' with an increase of only $0.001 \mathrm{~mm}$.

- Since the volume of the elements in the cross over points in the WiseTex/MeshTex model is higher than the overall thickness of the yarns, the total volume of these elements in the volume averaging technique will have an impact on the homogenized material property results for the stiffness in the main directions $E_{11}$ and $E_{22}$.

The mesh convergence between model 'B-M2-G2' and 'B-M4-G2' shows a decrease of the $\mathrm{E}_{33}$ of $0.09 \mathrm{GPa}$ due to the lower interpenetration of the mesh elements of the yarns and matrix. By comparing columns 6,7 and 8 in Table 4 , one notices that the homogenized macro elastic constants can be overestimated if the chosen PBC grid size is too coarse ('BM3-G3'). This is due to the higher leverage on the nodes in a PBC grid cell, because of the higher distance between the reference node on which the PBC constraint is put and the FE mesh node (Figure 7(i)).

Additionally a small study on the influence of the heart line mesh has been implemented on model 'B-M4-G2' (Figure 5). The mesh size of the heart line of the yarns could have an influence on the numerical properties if the directions of the yarns vary a lot, e.g. high crimp at the cross over points of the yarns. However this highly depends on the change in direction 
of the geometry of the yarn. A study was made implementing two distinct mesh sizes for the heart lines, mesh size heart $1=0.05 \mathrm{~mm}$ (149 elements) corresponding to the mesh size of the yarns, and mesh size heart ${ }_{2}=1 \mathrm{~mm}$ (7 elements). Mesh size heart ${ }_{2}$ will lead to a bigger amount of elements in a same section with identical material orientation than with mesh size heart $_{1}$ (Figure 13(b) and Figure 13(a) respectively). Since no high variations can be noticed in the yarns geometry (small crimp), the results on the global mechanical properties (Figure 13(c) $)($ E11 = 56.335 GPa; E22 = 56.333 GPa; E33 = 10.657 GPa; G12 = $4.403 \mathrm{GPa} ; \mathrm{G} 13=$ $3.216 \mathrm{GPa} ; \mathrm{G} 23=3.216 \mathrm{GPa} ; v 12=0.074 ; v 13=0.42 ; v 23=0.42)$ do not change much compared to column 'B-M4-G2' of Table 4.

\subsection{Comparison of the local strain field profiles}

In publications $[22,31,32]$ the importance of the prediction and evaluation of the local strain profiles and gradients is underlined, since it could contribute to a reliable prediction of damage initiation and strength of the material. The development of a FE unit cell model which is able to predict the strain fields as observed experimentally can be a challenging task. Especially obtaining corresponding values of the strains obtained numerically and experimentally is very difficult. In order to be able to check the order of magnitude of the FE results obtained using both methods (ORAS and WisTex/MeshTex), an optical fibre Bragg sensor with a resolution of $5 \mu \varepsilon$ was embedded in the CETEX material with a total measuring length of $8 \mathrm{~mm}$ which overlaps the total length of a RUC (Figure 9 (b)). For a global applied average strain of $0.2 \%$, a maximum local measured strain was observed of $0.25 \%$ with a minimum of $0.16 \%$. These values of the strains are averaged values over a certain length (induced by the precision of the Bragg sensor) but the exact value of this length cannot be determined. Therefore a sensitivity analysis regarding the path over which the local strains are taken in the FE models is added in this section. The complete experimental process and results can be found in [22]. An overview of the results over multiple paths in the width of the RUC for the model using the new ORAS software, the state of the art approach WiseTex /MeshTex and a comparison between both models for a same chosen path of the RUC can be 
found in Figure 14 respectively Figure 15 and Figure 16. Figure 14 and Figure 16 show that the maximal local strain calculated using ORAS $(0.36 \%)$ is higher than the one obtained with the WiseTex/MeshTex model $(0.28 \%)$. A reason for this can be: i) the coarser mesh in the MeshTex model (37800 elements for the matrix and 15400 elements for the yarns) compared to the ORAS matrix mesh $(1,078,427$ elements for the matrix and 133,266 elements for the yarns) and in order to capture the rotations of the solid elements of the yarns correctly, a sufficient amount of elements through the thickness of the yarns should be used since the solid elements have no rotational degrees of freedom (Figure 4), ii) the difference in thickness at the cross over points of the yarns of the WiseTex/MeshTex model (Figure 4(e)(f)). The minimal local strain obtained with the ORAS model $(0.16 \%)$ is also slightly higher than the one obtained with the MeshTex model $(0.14 \%)$ and corresponds to the value obtained experimentally $(0.16 \%)$. If one compares the maximum and minimum local strains of the FE models with the experimentally obtained values, one can see that the order of magnitude is correct for both methods, but the maximum obtained with the ORAS model is higher. But when averaging the value over $1 \mathrm{~mm}$ around the peak values, the local strain of the ORAS model equals $0.25 \%$ and the same value is obtained for the MeshTex model and both correspond to the value seen by the Bragg sensor. Additionally, since the position of the path on which the strains are extracted in the FE models influences highly the minimum and maximum local strain values, and the exact position of the Bragg sensor in the RUC could not be extracted (undulation of the Bragg sensor, parallelism of the sensor with the yarns in the Ydirection...), only the order of magnitude can be predicted. In order to be able to validate both FE models, the WiseTex/MeshTex model and the ORAS model, with an embedded fibre Bragg sensor, this sensor should explicitly be implemented, with a correct position in the RUC and similar cross section, in the FE models, with the correct position of the Bragg sensor and yarns obtained through a micro-CT scan. This could be achieved with the ORAS software and is future work. 
3.5. Implementation of periodic boundary conditions on a complex geometry, a spread tow reinforced composite, using the new technique

The aim of this section is to show the possibilities of the new PBC implementation technique with the construction of a RUC of a spread tow plain weave fabric reinforced composite and the FE calculation of the model. A wide spread $(15-20 \mathrm{~mm})$ and thin $(0.05-0.1 \mathrm{~mm})$ yarn, also called spread tow, is woven into a plain weave fabric with one of the big advantages that the mechanical properties compared to regular tows are increased because of the reduced crimp of the tow. A RUC of a carbon fibre spread tow plain weave reinforced composite is built in a CAD software and after meshing in a commercial FE software, the PBC were applied using the new technique. The big difficulty for the existing meso-scale software products is to achieve the fibre volume fractions as obtained experimentally [33]. For example an average of $50.5 \%$ fibre volume fraction in a spread tow (width $=20 \mathrm{~mm}$, height $=0.05 \mathrm{~mm}$ ) with an overall composite fibre volume fraction of $50.4 \%$ has to be obtained. This requires a very small amount of matrix in between the spread tows. Using the new technique described in this paper, one obtains an overall composite fibre volume fraction of 50.27\% (with 50.5\% of fibre volume fraction in the spread tows) with very small pockets of the matrix (Figure 17(a)) and the results of a FE calculation shown in Figure 17(b).

\section{Conclusions}

A method for the construction of meso-scale FE models of textile reinforced composites using periodic boundary conditions on multiple part meshes (ORAS) has been developed and defined. The technique has been validated by comparison to experimental results and to the state of the art validated models (WisTex/MeshTex) for a $5 \mathrm{H}$-satin weave unit cell. This new technique opens new paths for the research of complex meso-scale architectures of textile composites.

\section{Acknowledgements}

The authors would like to acknowledge TenCate for supplying the composite materials.

\section{References}

[1] Stepan V Lomov and et al, "Meso-FE modelling of textile composites: Road map, data 
flow and algorithms," Composites Science and Technology, vol. 67, no. 9, pp. 18701891, 2007.

[2] Brian N Cox and Gerry Flanagan, Handbook of Analytical Methods for Textile Composites. Hampton: National Aeronautics and Space Administration, Langley Research Center, 1997.

[3] Christopher M Pastore, "Opportunities and Challanges for Textile Reinforced Composites," Mechanics of Composite Materials, vol. 36, no. 2, pp. 97-116, 2000.

[4] A E Bogdanovich and Christopher M Pastore, Mechanics of Textile and Laminated Composites. London: Chapman \& Hall, 1996.

[5] J Kabelka, "Prediction of the Thermal Properties of Fibre-Resin Composites," in Developments in Reinforced Plastics. London: Elsevier, 1984, vol. 3, pp. 167-202.

[6] J Whitcomb, K Woo, and S Gundapaneni, "Macro Finite Element Analysis of Textile Composites," Proc. 6th Japan-U.S. Conference on Composite Materials, pp. 541-550, 1992.

[7] R V Marrey and B V Sankar, "A Micromechanical Model for Textile Composite Plates," Journal of Composite Materials, vol. 31, no. 12, pp. 1187-1213, 1997.

[8] T Yoshino and T Ohtsuka, "Inner Stress-Analysis of Plane Woven Fiber Reinforced Plastic Laminates," Bulletin of the JSME-Japan Society of Mechanical Engineers, vol. 25, no. 202, pp. 485-492, 1982.

[9] John D Whitcomb, "Three-Dimensional Stress Analysis of Plain Weave Composites," NASA - Technical Memorandum - 101672, 1989.

[10] A Dasgupta and S M Bhandarkar, "Effective Thermomechanical Behavior of PlainWeave Fabric-Reinforced Composites Using Homogenization Theory," Journal of Engineering Materials and Technology, vol. 116, pp. 99-105, 1994.

[11] N K Naik and V K Ganesh, "Prediction of On-Axes Elastic Properties of Plain Weave Fabric Composites," Composites Science and Technology, vol. 45, pp. 135-152, 1992.

[12] P Paumelle, A Hassim, and F Lene, "Microstress Analysis in Woven Composite Structure," Recherche Aerospatiale, no. 6, pp. 47-62, 1991.

[13] D M Blackketter, D E Walrath, and A C Hansen, "Modeling Damage in a Plain Weave Fabric-Reinforced Composite-Material," Journal of Composite Technology \& Research, vol. 15, no. 2, pp. 136-142, 1993.

[14] E H Glaessgen, C M Pastore, O H Griffin, and A Birger, "Geometrical and Finite Element Modelling of Textile Composites," Composites: Part B, vol. 27B, pp. 43-50, 1996.

[15] R Mcllhagger, B J Hill, D Brown, and L Limmer, "Construction and analysis of threedimensional woven composite materials," Composites Engineering, vol. 5, no. 9, pp. 1187-1197, 1995.

[16] SV Lomov, X Ding, S Hirosawa, and et al., "FE simulations of textile composites on unit cell level: validation with full-field strain measurements.," in Proc SAMPE Europe International Conference, 2005, pp. 28-33.

[17] I Verpoest and SV Lomov, "Virtual textile composites software WiseTex: integration with micro-mechanical, permeability and structural analysis.," Composites Science and Technology, vol. 65, no. 15-16, pp. 2563-74, 2005.

[18] T Kurashiki, M Zako, S Hirosawa, SV Lomov, and I Verpoest, "Estimation of a mechanical characterization for woven fabric composites by fem based on damage mechanics," in ECCM-11, 11th European Conference on Composite Materials, 2004. 
[19] Peter Linde, Peter Middendorf, Björn Van Den Broucke, and Henk De Boer, "Numerical Simulation of Damage Behaviour of Textile Reinforced Composites in Aircraft Structures," in ICAS, Nice (France), 2010.

[20] Zheng Yuan and Jacob Fish, "Toward realization of computational homogenization in practice," Int. J. Numer. Meth. Engng, vol. 73, pp. 361-380, 2008.

[21] V.-D. Ngyuen, E Béchet, C Geuzaine, and L Noels, "Imposing periodic boundary condition on arbitrary meshes by polynomial interpolation," Computational Materials Science, vol. 55, pp. 390-406, 2012.

[22] Subbareddy Daggumati, Concurrent Modelling and Experimental Analysis of MesoScale Strain Fields and Damage in Woven Composites under Static and Fatigue Tensile Loading. Ghent: Ghent University, 2011, PhD Thesis - ISBN 9789085784258, https://biblio.ugent.be/publication/3259370.

[23] C.C. Chamis, "Mechanics of Composite Materials: Past, Present and Future," NASA Technical Memorandum, vol. 100793, 1984.

[24] TexGen. texgen.sourceforge.net.

[Online]. http://texgen.sourceforge.net/index.php/Main_Page

[25] Norris F Dow, V Ramnath, and B Walter Rosen, "Analysis of Woven Fabrics for Reinforced Composite Materials," National Aeronautics and Space Administration, Hampton, Technical report 1987.

[26] V Carvelli and C Poggi, "A homogenization procedure for the numerical analysis of woven fabric composites," Composites: Part A, vol. 32, pp. 1425-1432, 2001.

[27] John Whitcomb, Kanthikannan Srirengan, and Clinton Chapman, "Evaluation of homogenization for global/local stress analysis of textile composites," Composite Structures, vol. 31, pp. 137-149, 1995.

[28] Jacob Aboudi, Marek-Jerzy Pindera, and Steven M Arnold, "Higher-order theory for periodic multiphase materials with inelastic phases," International Journal of Plasticity, vol. 19, pp. 805-847, 2003.

[29] James R Bunch and John E Hopcroft, "Triangular Factorization and Inversion by Fast Matrix Multiplication," Mathematics of Computation, vol. 28, no. 125, pp. 231-236, 1974.

[30] Ives De Baere, Experimental and Numerical Study of Different Setups for Conducting and Monitoring Fatigue Experiments of Fibre-Reinforced Thermoplastics. Ghent: Ghent University, 2008, PhD Thesis, ISBN: 9789085781967, https://biblio.ugent.be/publication/470995.

[31] P Potluri, R J Young, K Rashed, A Manan, and Y T Shyng, "Meso-scale strain mapping in UD woven composites," Composites: Part A, vol. 40, pp. 1838-1845, 2009.

[32] S V Lomov et al., "Full-field strain measurements for validation of meso-FE analysis of textile composites," Composites: Part A, vol. 39, pp. 1218-1231, 2008.

[33] Yasuhiro Nishikawa, Kazuya Okubo, Toru Fujii, and Kazumasa Kawabe, "Fatigue crack constraint in plain-woven CFRP using newly developed spread tows," International Journal of Fatigue, vol. 28, pp. 1248-1253, 2006.

\section{Tables captions}

Table 1: Material properties of the 5H-Satin weave carbon fibre-PPS composite obtained through a micro-CT scan and from the Tencate Datasheet for the CF/PPS 5H-Satin weave composite 
Table 2: Periodic boundary conditions for the RUC

Table 3: Fibre volume fractions for the FE RUC models

Table 4: Validation of the FE calculation of the elastic constants of a 5H-satin Weave carbonPPS composite

\section{Figures captions}

Figure 1: Micro-CT analysis of a 5H-satin weave composite CF/PPS composite

Figure 2: Geometric model of the 5H-satin weave RUC created with a CAD software.

Figure 3: CAD model creation of a yarn starting from a cross section and a heart line

Figure 4: (a) and (b) 3D FE mesh of the matrix for 'Model A' and 'Model B'; (c) 3D fine FE mesh of the yarns with thickness $0.156 \mathrm{~mm}$; (d) 3D coarse FE mesh of the yarn with thickness $0.155 \mathrm{~mm}$ and $0.156 \mathrm{~mm}$; (e) 3D FE mesh in MeshTex of a WiseTex generated model; (f) influence of the MeshTex algorithm on the thickness variation of a yarn with the local influence on the yarn cross sectional shape

Figure 5: Schematic tree explaining the nomenclature of the different models used, going from the CAD model to the FE mesh model to the calculated FE model + PBC Grid size.

Figure 6: (a) Presentation of the C\# code for the creation of the local material orientation of the yarn; (b) Grid creation of a domain; (c) Redistribution of a floating node

Figure 7: (a)(b)(c) FE results for the calculations using the MeshTex model with PBC implementation using the new methodology (a) (b) and using the state of the art implementation (c); (d)(e)(f)(g) FE results for the calculations using the models created using a CAD software with PBC implementation using the new methodology and with same PBC grid size (G2) with coarse mesh (d)(f) and fine mesh (e)(f); (h)(i) ) FE results for the calculations using the models created using a CAD software with PBC implementation using the new methodology with fine mesh and PBC grid size $=0.01 \mathrm{~mm}(\mathrm{~h})$ and $\mathrm{PBC}$ grid size $=$ $0.2 \mathrm{~mm}$ (i)

Figure 8: (a) Partitioning of a model with release of the tie constraints; (b) Erasing overconstrained nodes in the PBC equations and keeping the tie constraints

Figure 9: Experimental test samples with (a) strain gauges and (b) an embedded fibre Bragg sensor

Figure 10: Experimental results for the stress - strain curves for (a) the longitudinal strain $\left(\varepsilon_{\mathrm{xx}}\right)$ and (b) the transverse strain $\left(\varepsilon_{\mathrm{yy}}\right)$

Figure 11: Experimental results for (a) the in-plane shear strain $\left(\tau_{\mathrm{xy}}\right)$ stress - strain curve and (b) the Poisson's ratio $\left(v_{12}\right)$ as function of the longitudinal strain $\left(\varepsilon_{\mathrm{xx}}\right)$

Figure 12: Influence of the mesh size on the fibre volume fraction

Figure 13: Influence of the mesh size of the heart lines on the homogenized elastic properties of the $5 \mathrm{H}$-satin weave $\mathrm{CF} / \mathrm{PPS}$ composite

Figure 14: Analysis of the local strain contours with additional position sensitivity analysis for the ORAS model 
Figure 15: Analysis of the local strain contours with additional position sensitivity analysis for the WiseTex/MeshTex model

Figure 16: Comparative analysis of the local strain contours of the ORAS model and the WiseTex/MeshTex model over the same path of the RUC

Figure 17: (a) CAD model of a spread tow woven fabric reinforced RUC; (b) FE mesh and calculation of a spread tow woven fabric reinforced RUC with PBC 
Figures

(a)

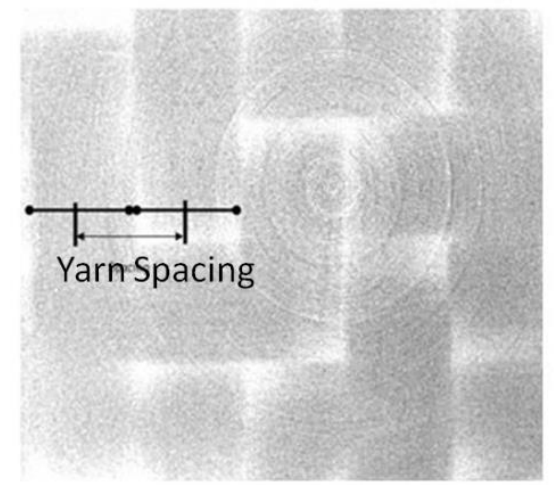

(c)

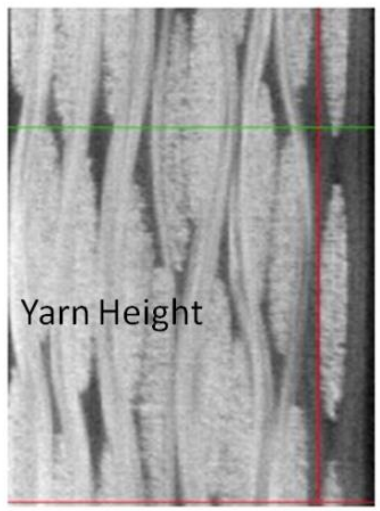

(b)

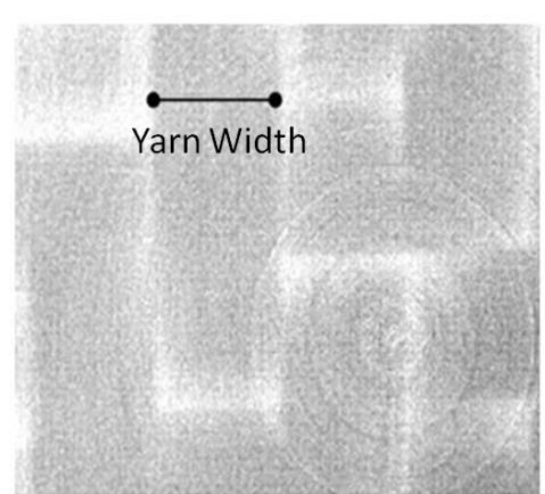

(d)

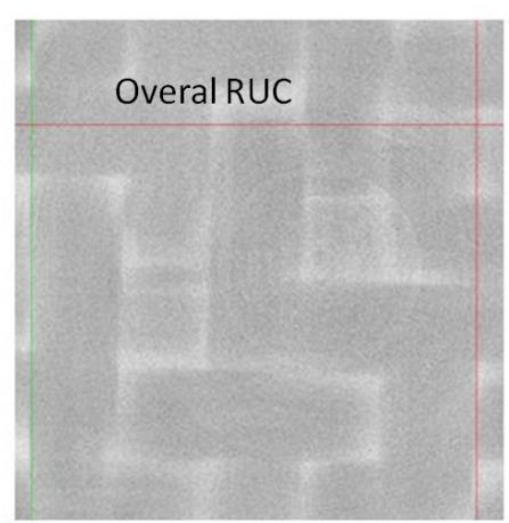

Figure 1

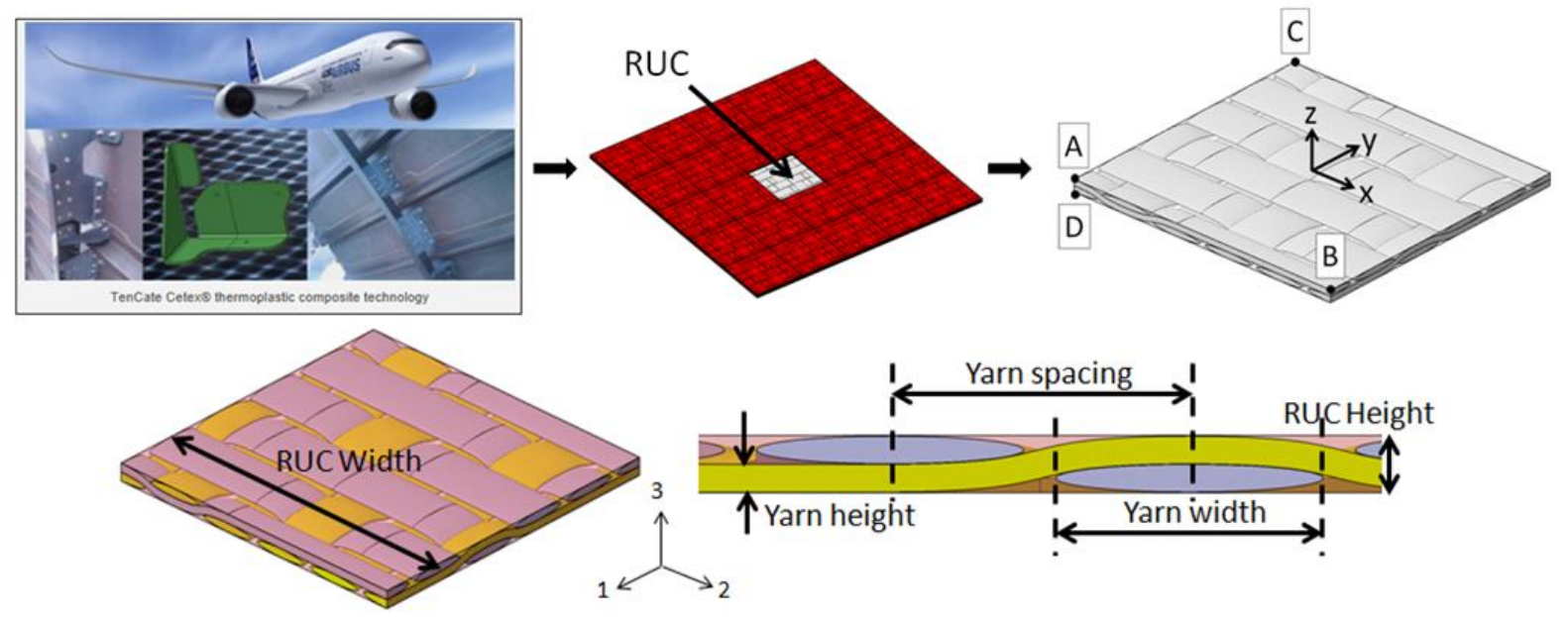

Figure 2 


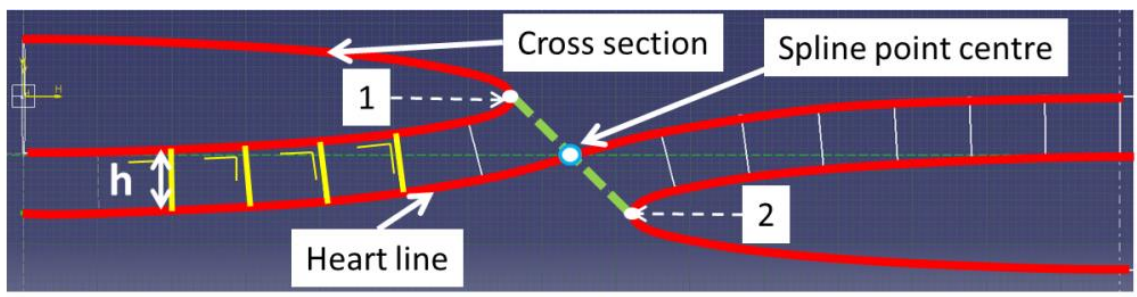

(a)

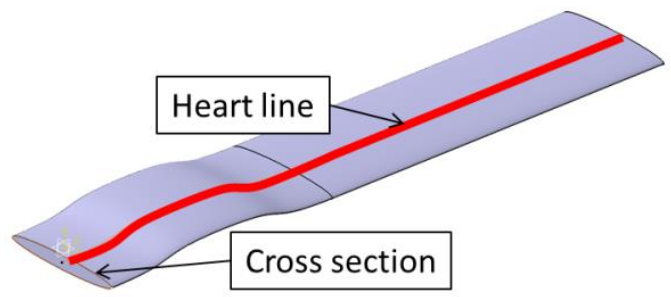

(b)

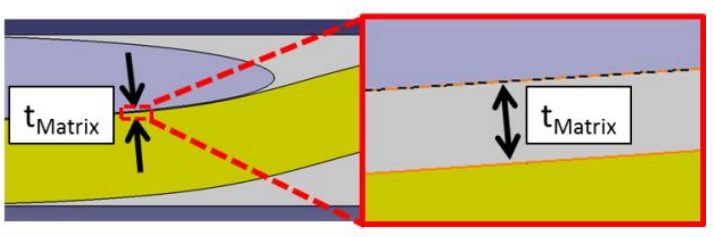

(c)

Figure 3

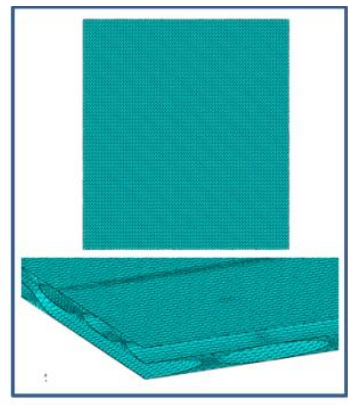

(a)

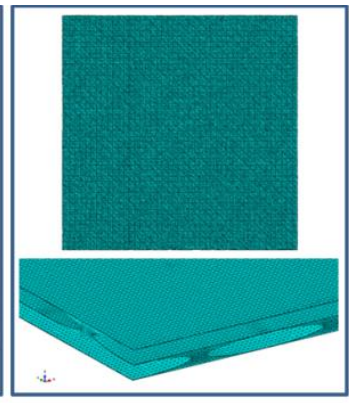

(b)

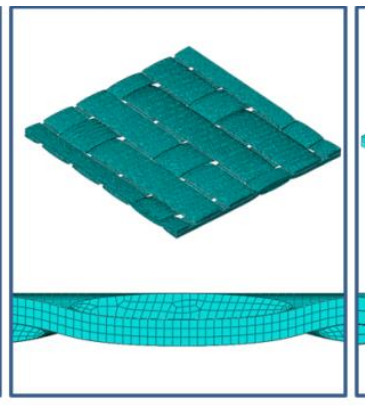

(c)

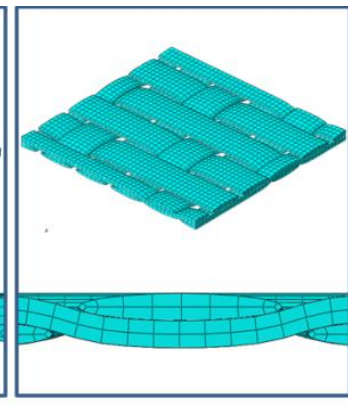

(d)

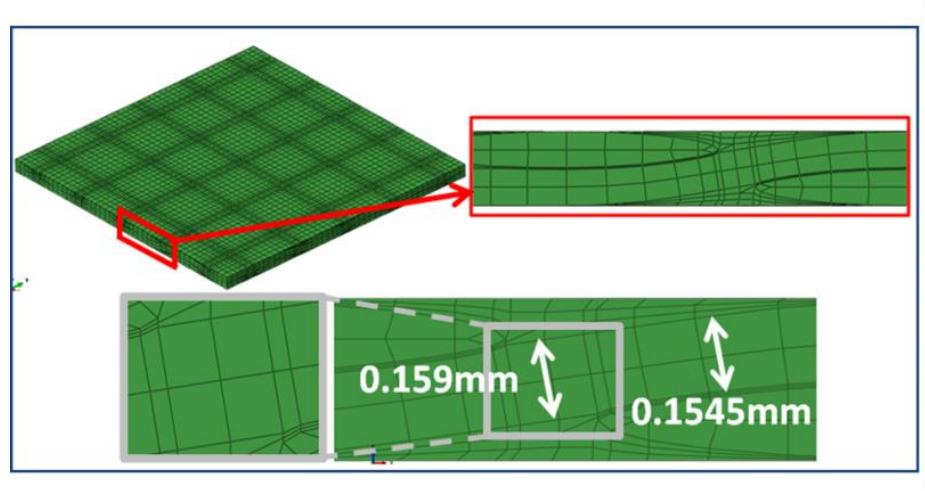

(e)

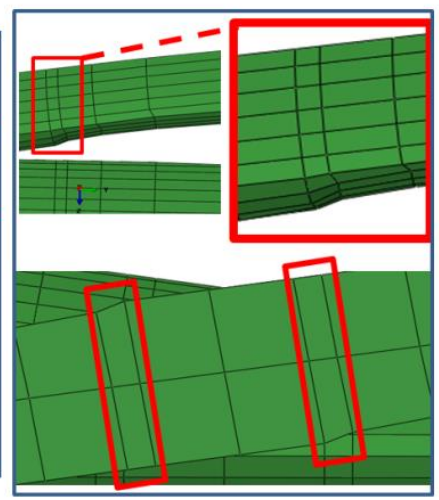

(f)

Figure 4 


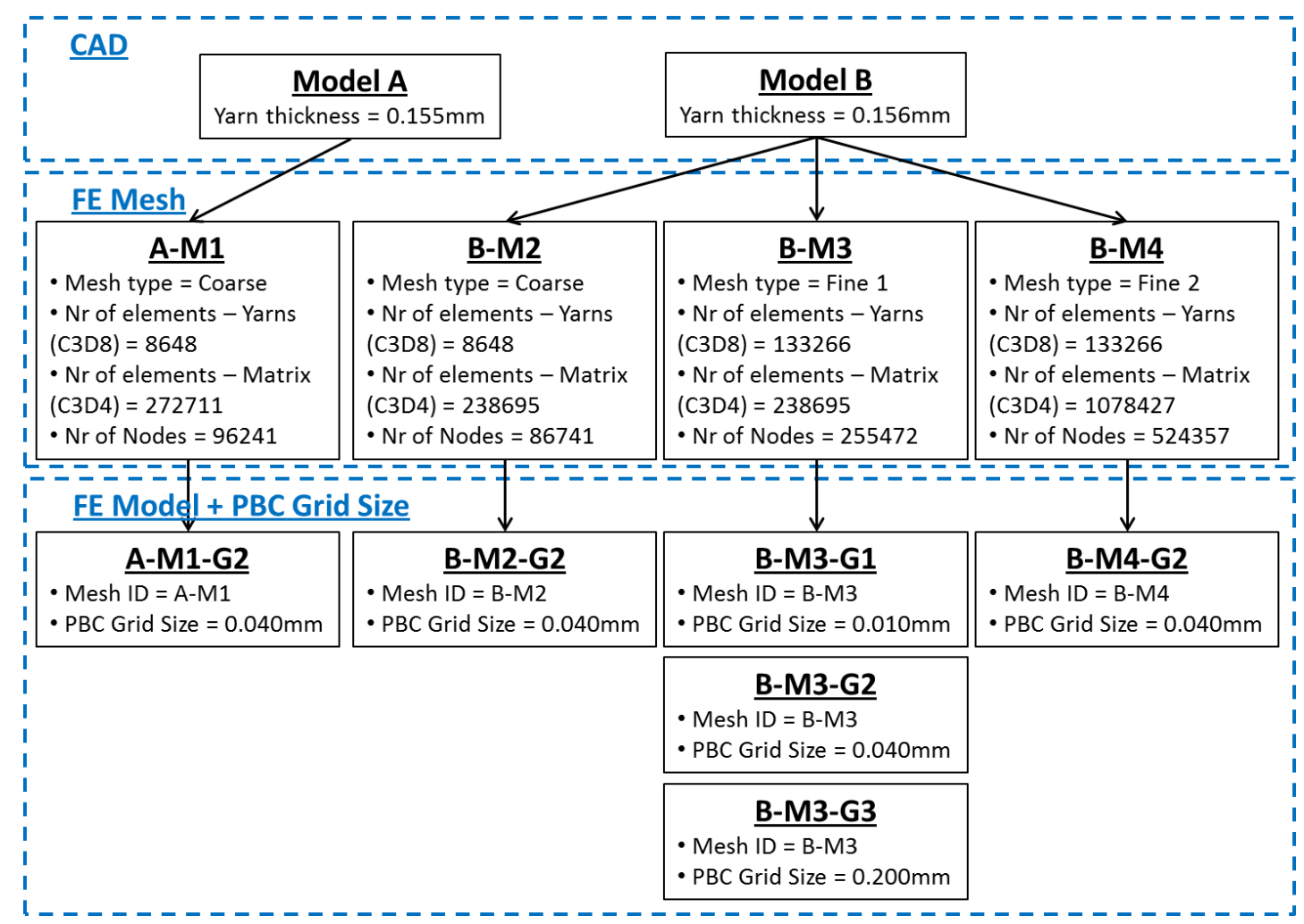

Figure 5

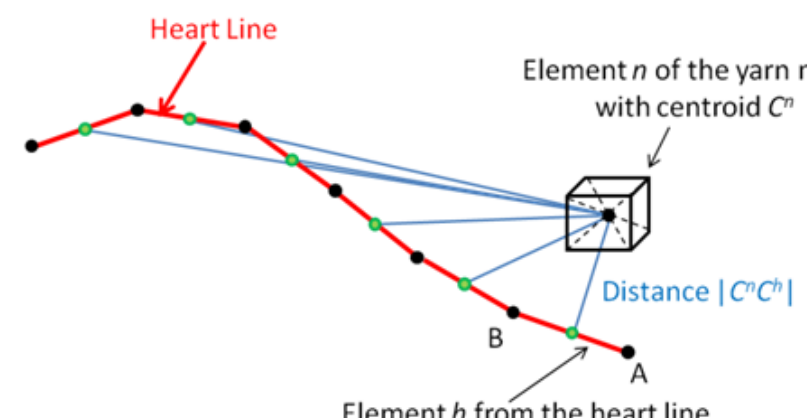

Element $h$ from the heart line mesh with centroid $C^{h}$

(a)

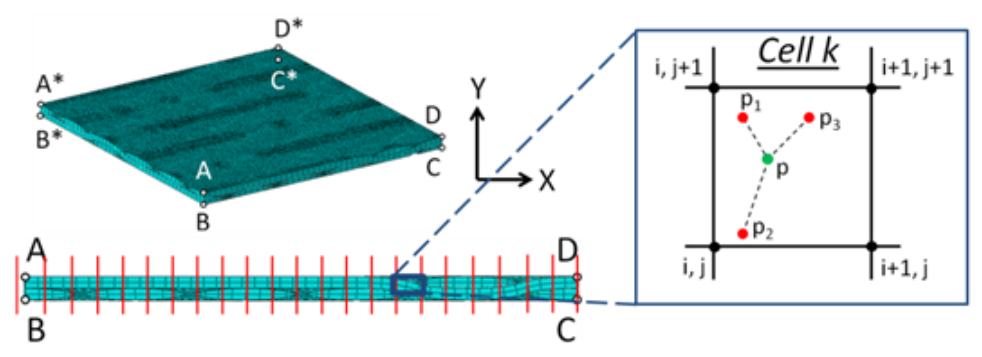

(b)

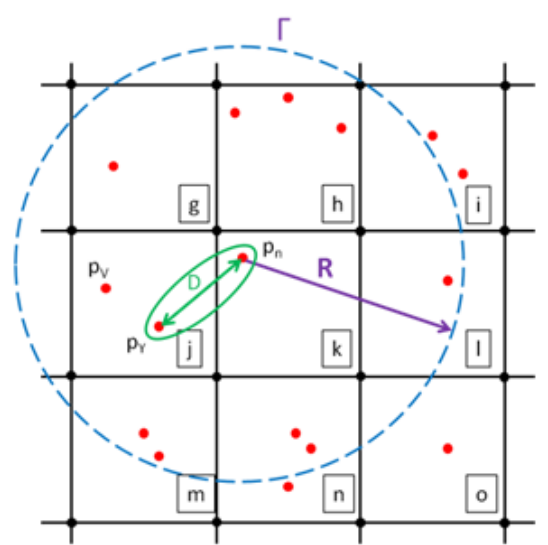

(c)

Figure 6 


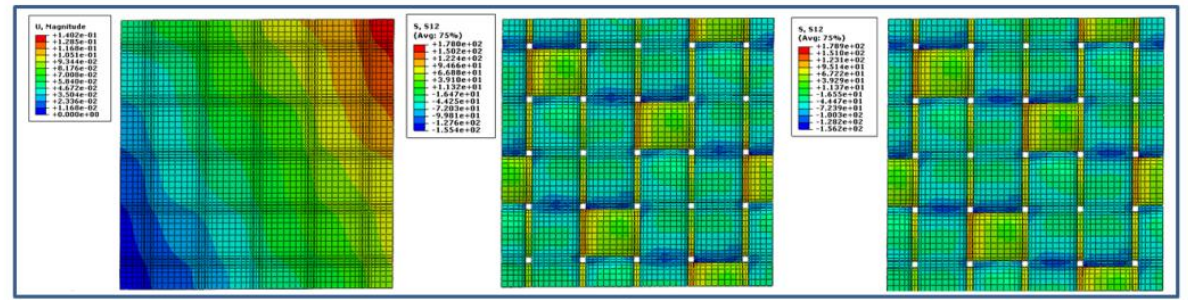

(a)

(b)

(c)
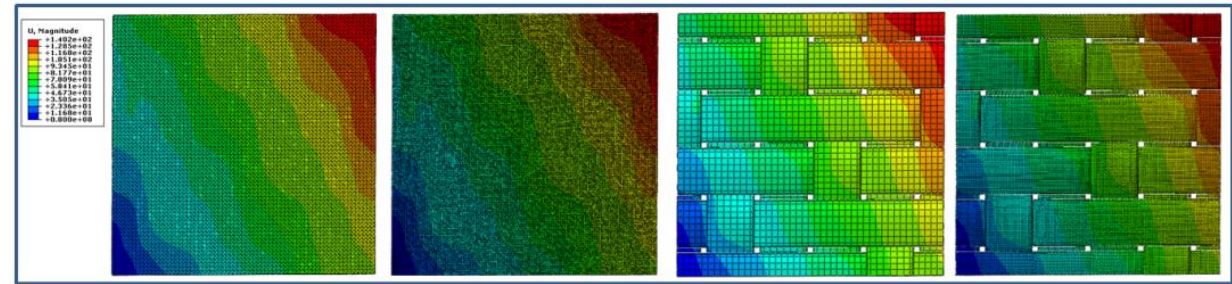

(d)

(e)

(f)

(g)

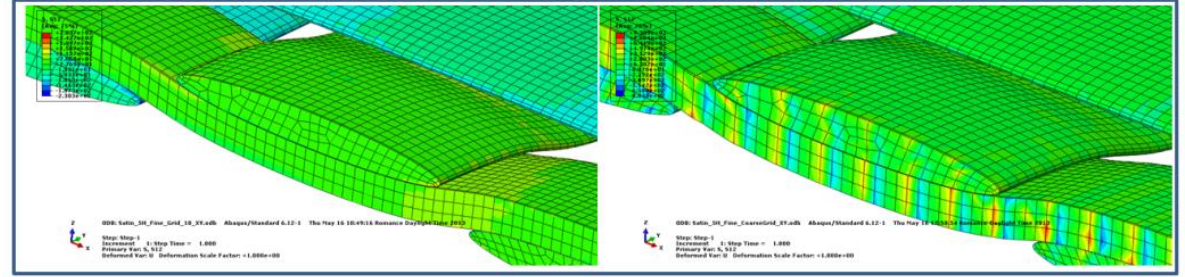

(h)

(i)

Figure 7
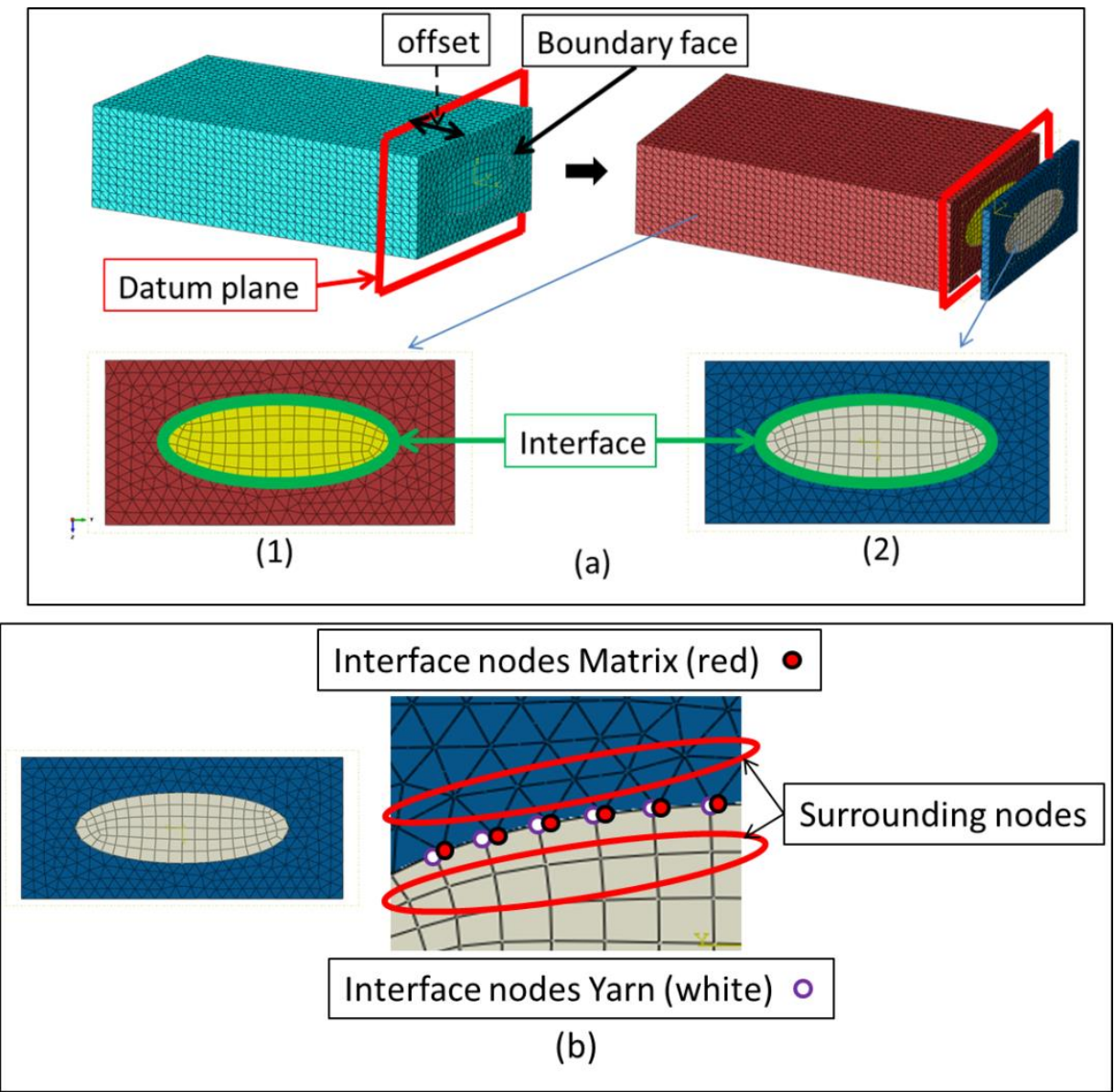

Figure 8 


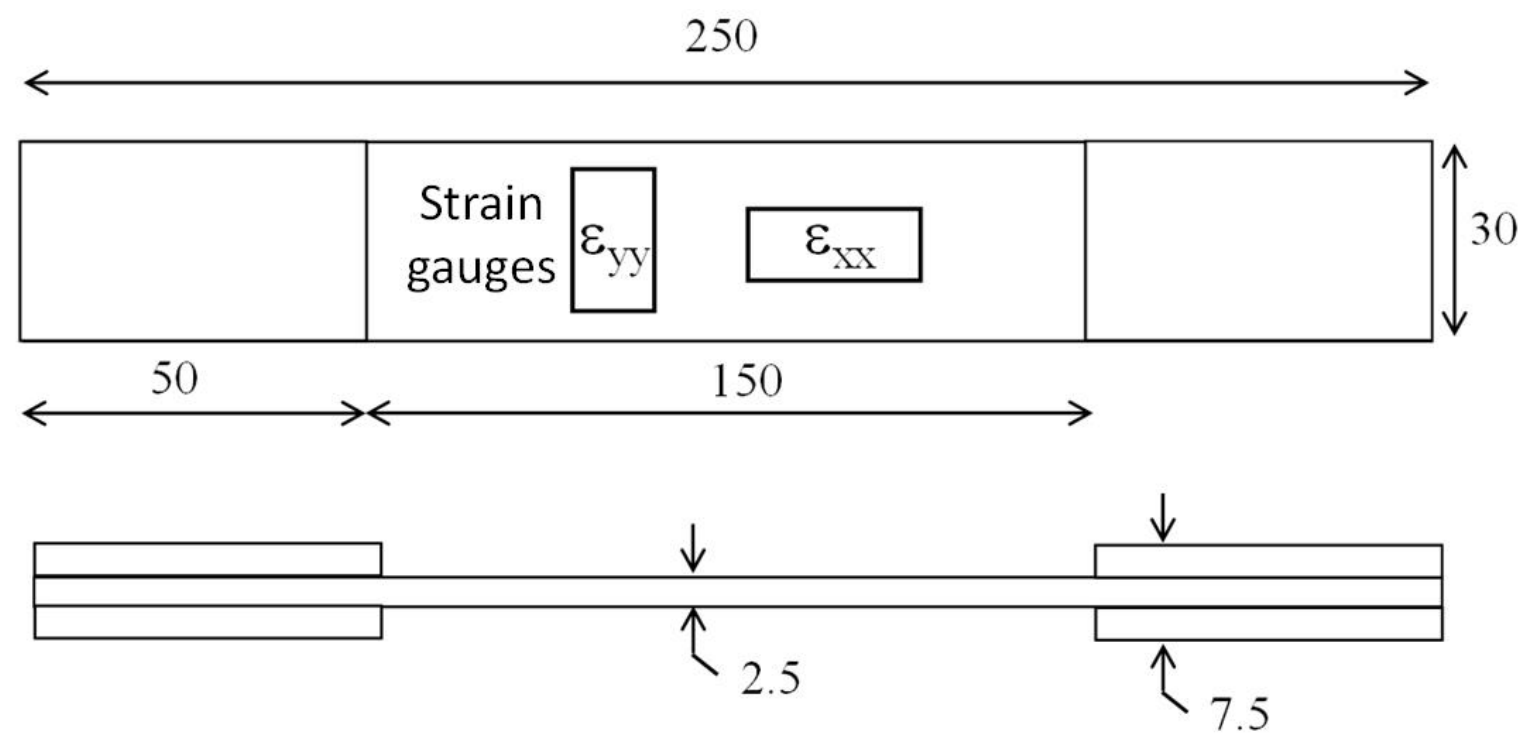

(a)
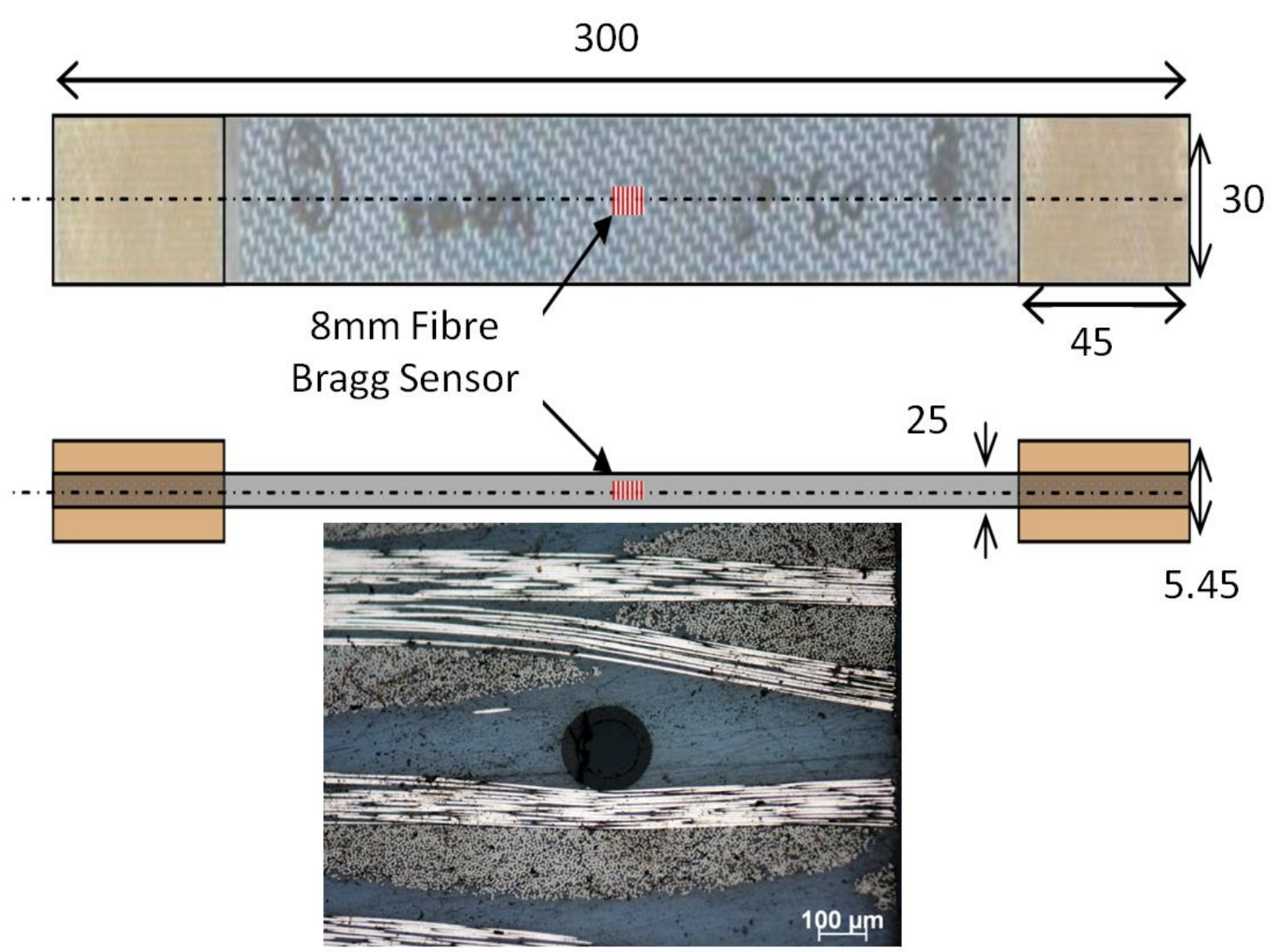

(b)

Figure 9 
Figure 2

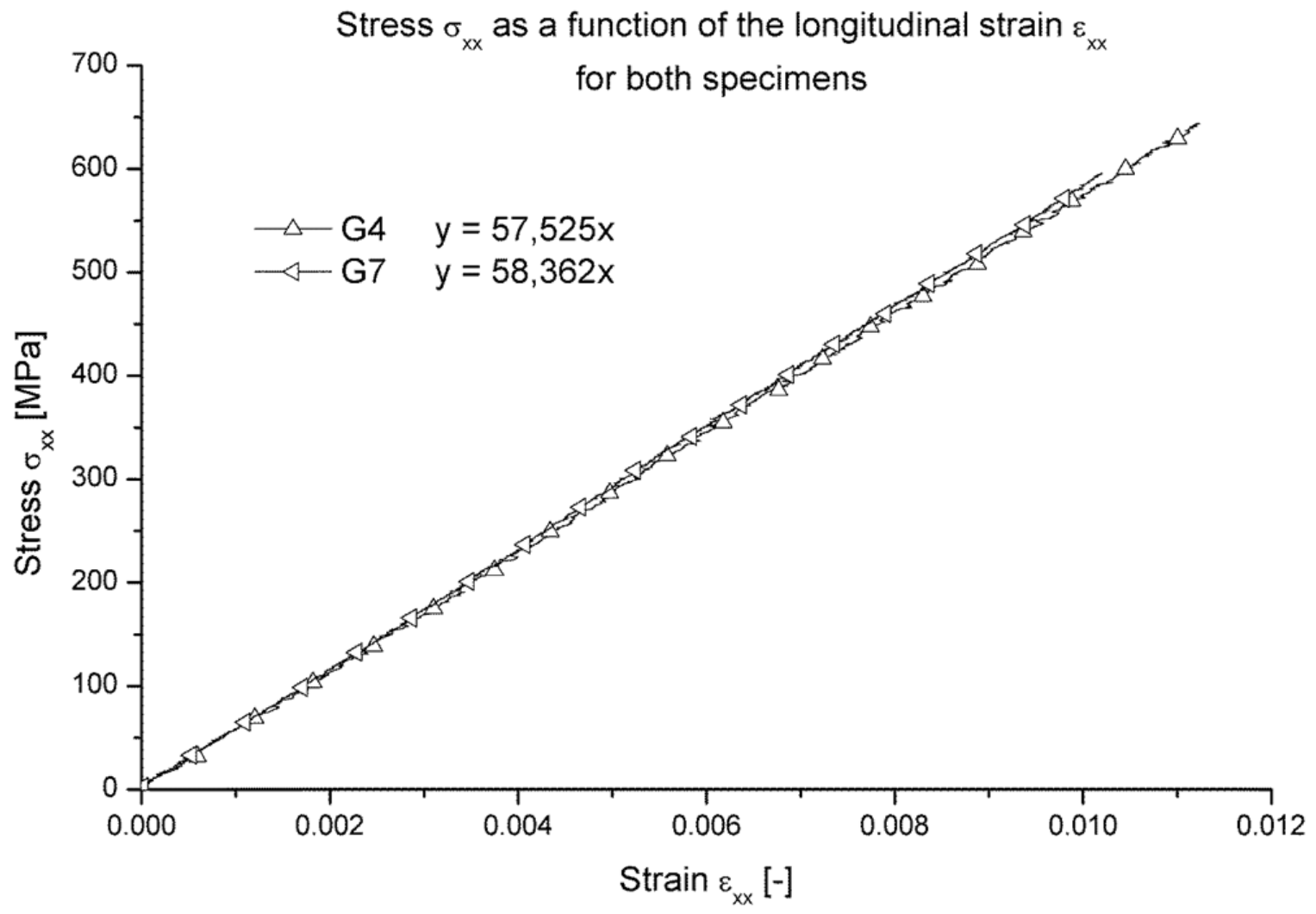

(a)

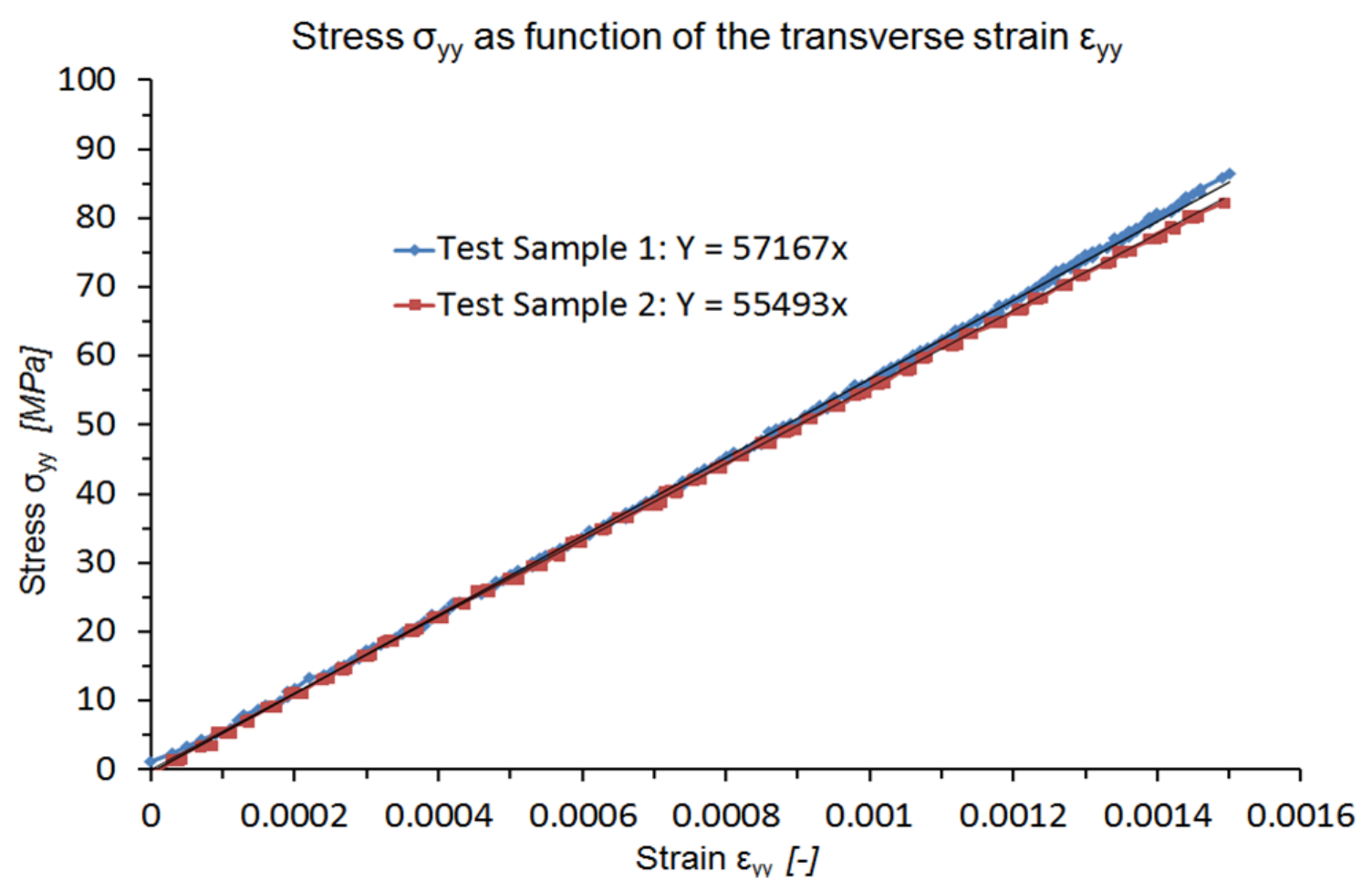

(b)

Figure 10 


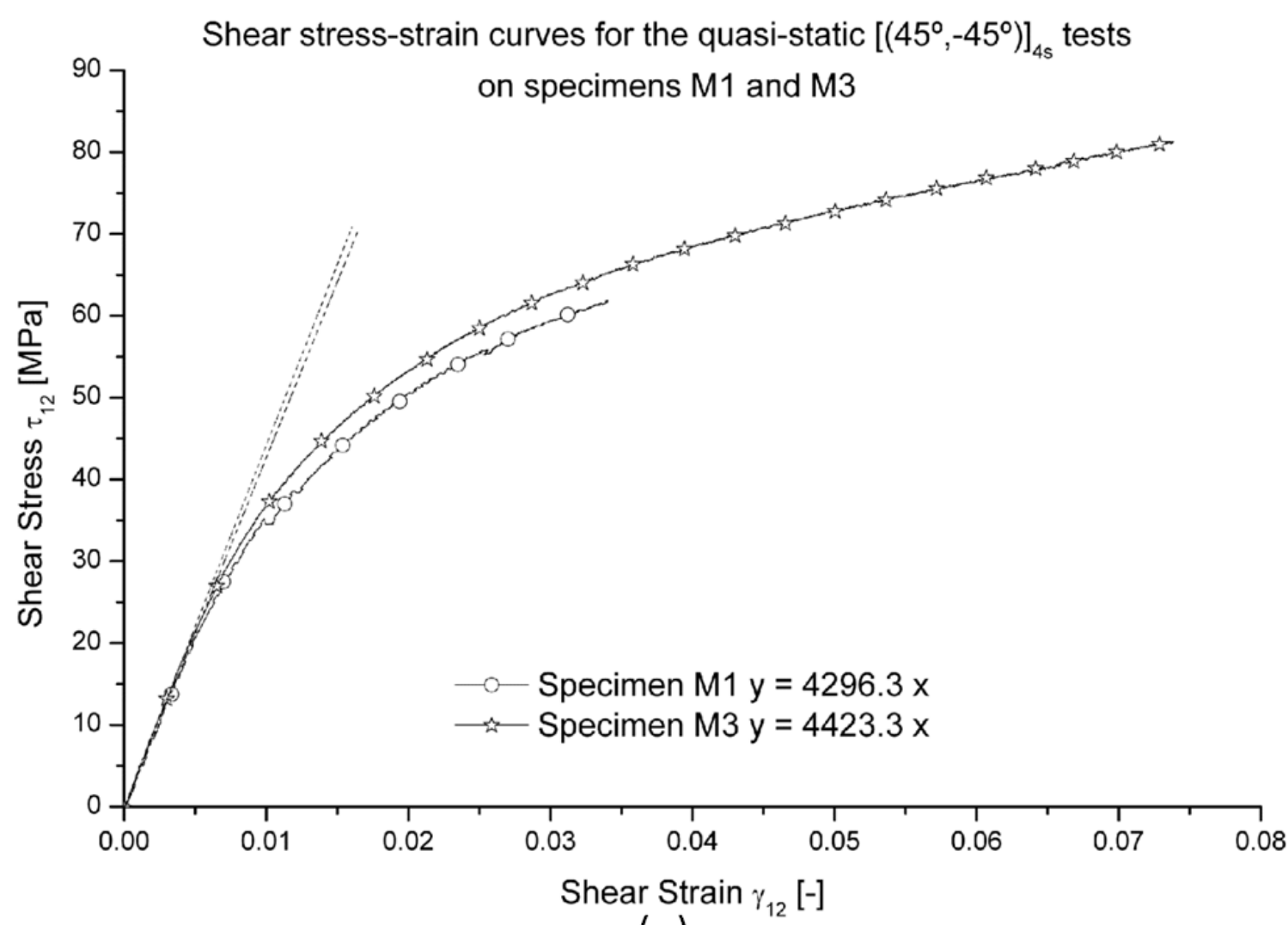

(a)

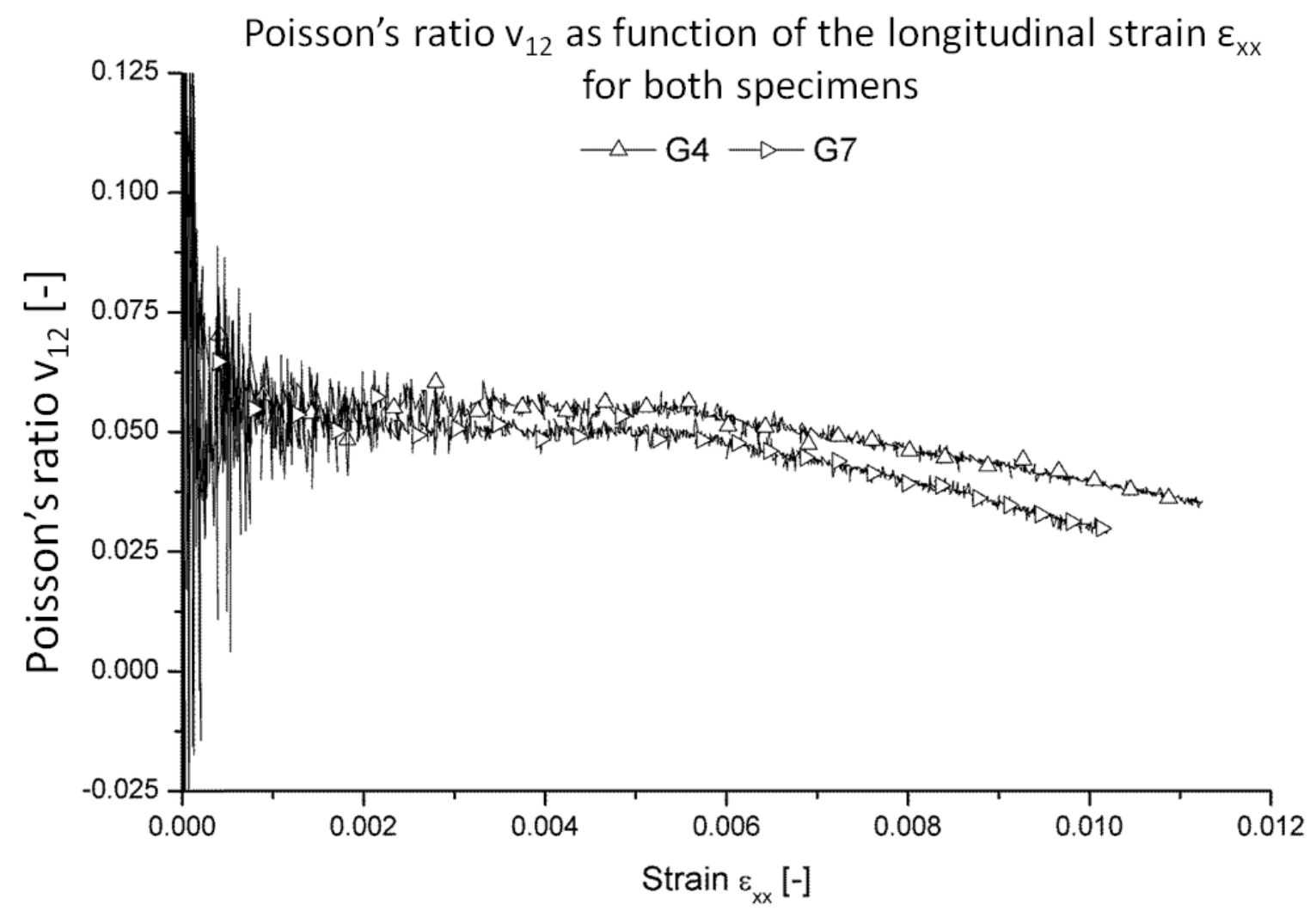

(b)

Figure 11 


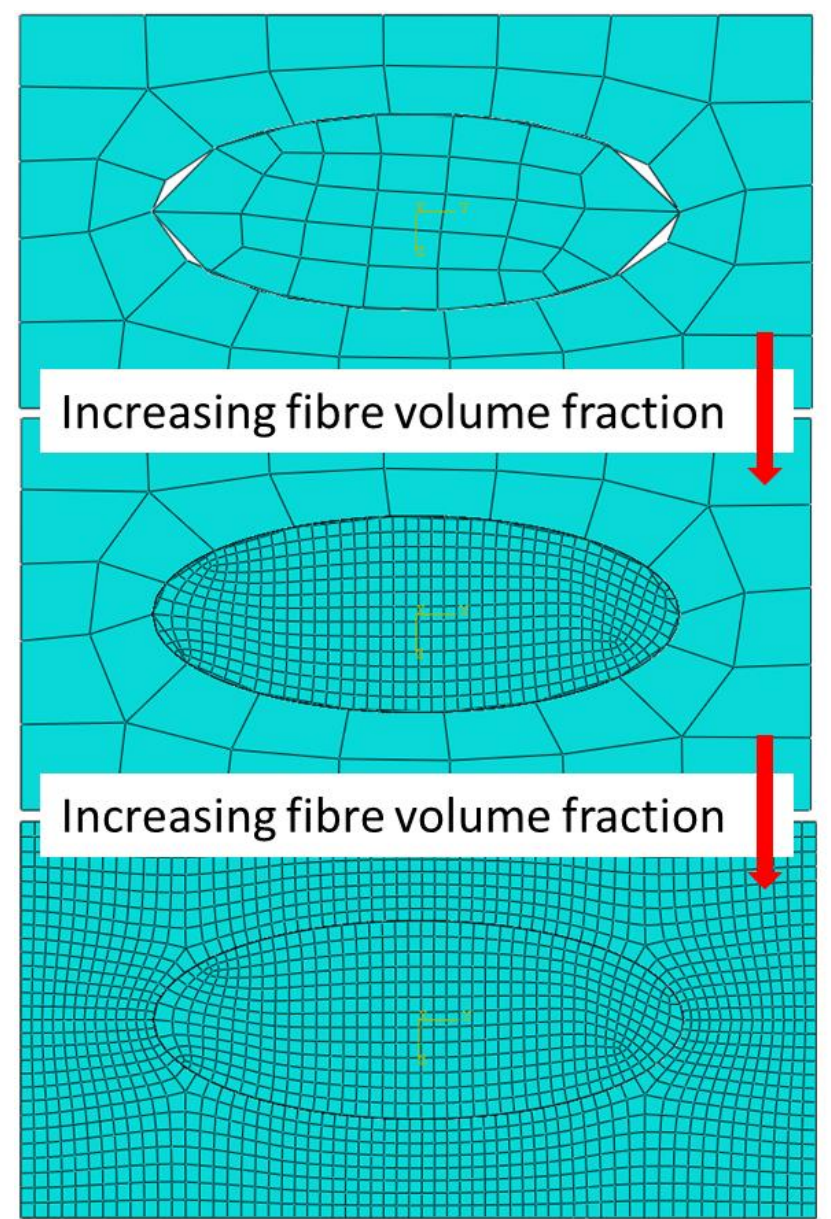

Figure 12

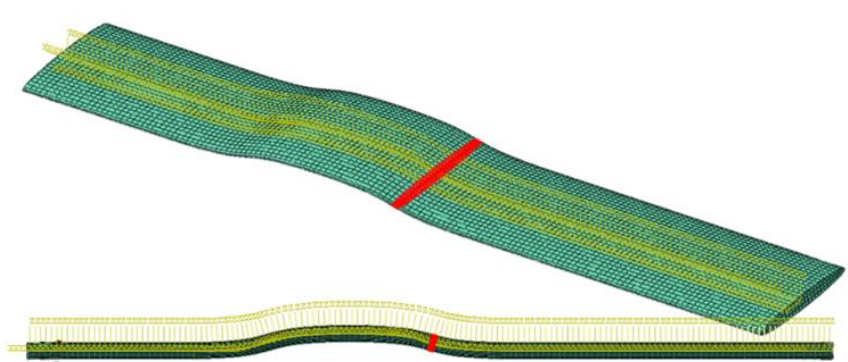

(a)

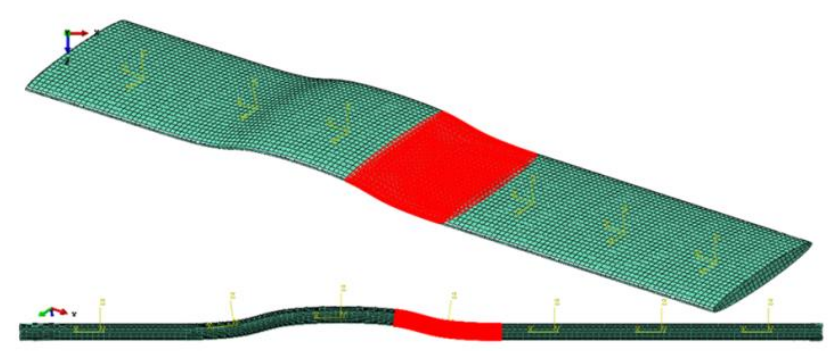

(b)
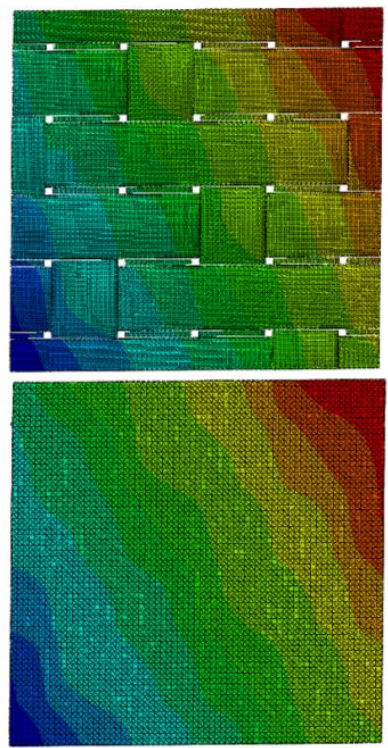

(c)

Figure 13 


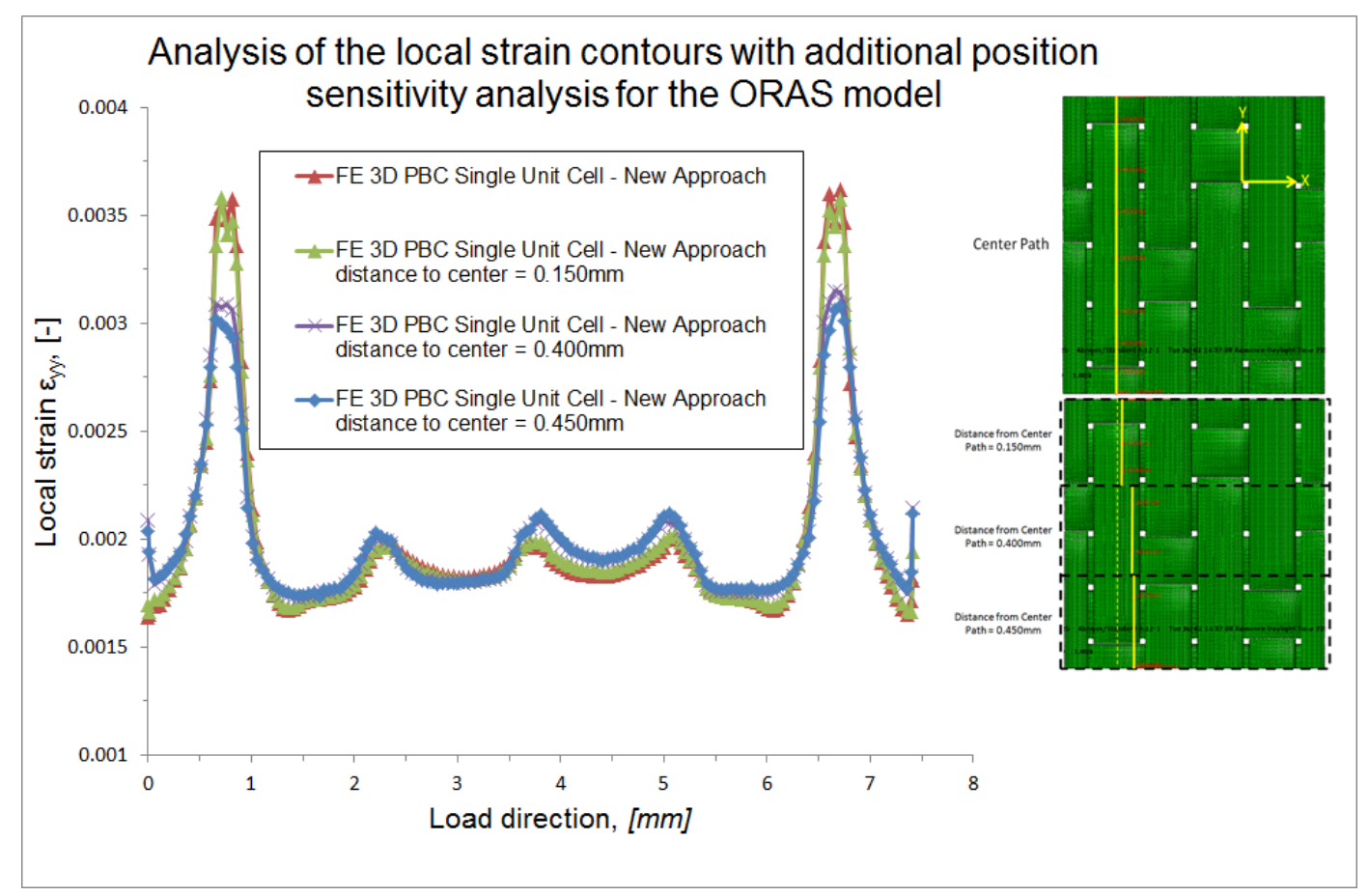

Figure 14

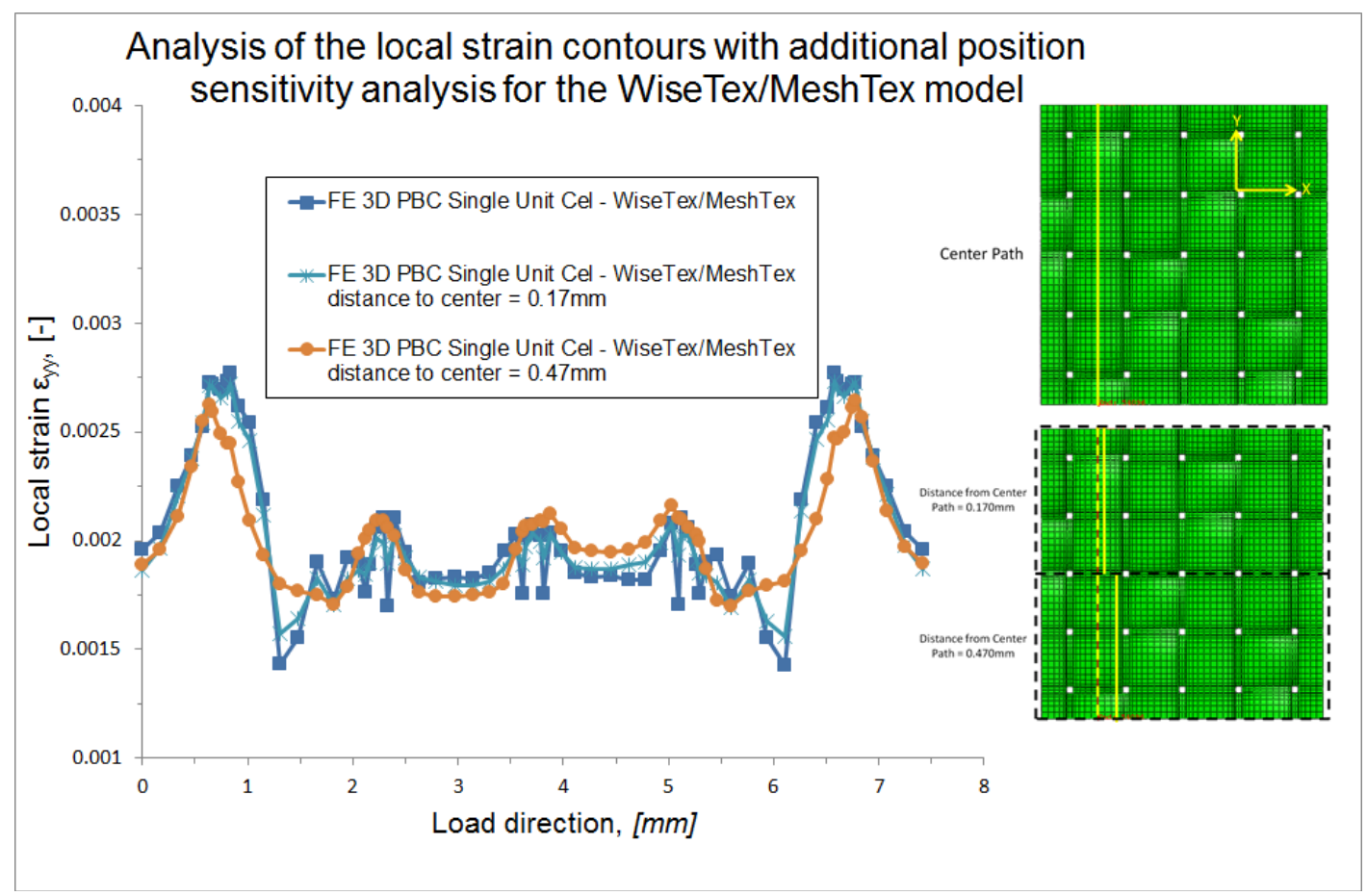

Figure 15 


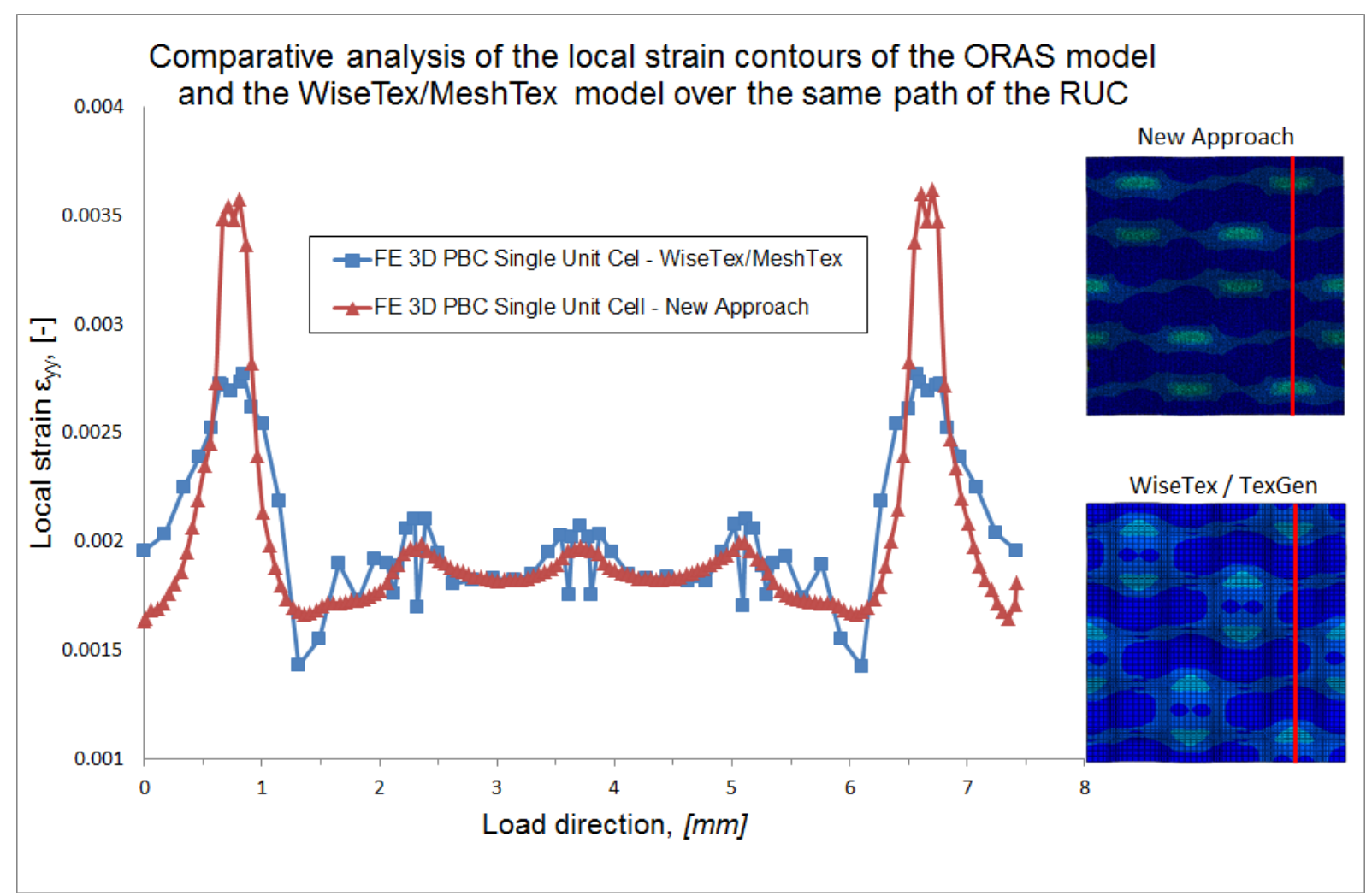

Figure 16

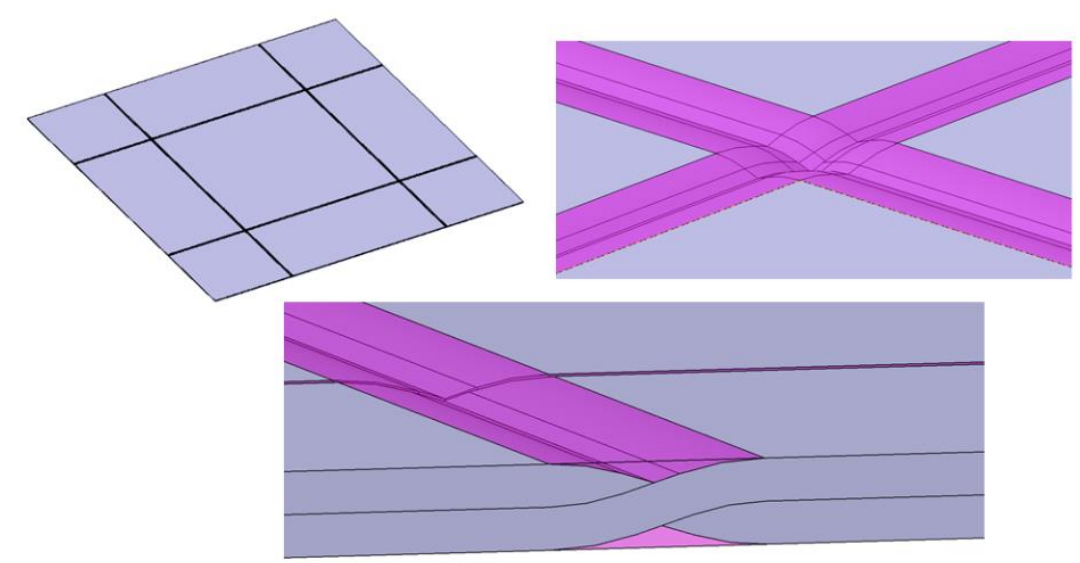

(a)

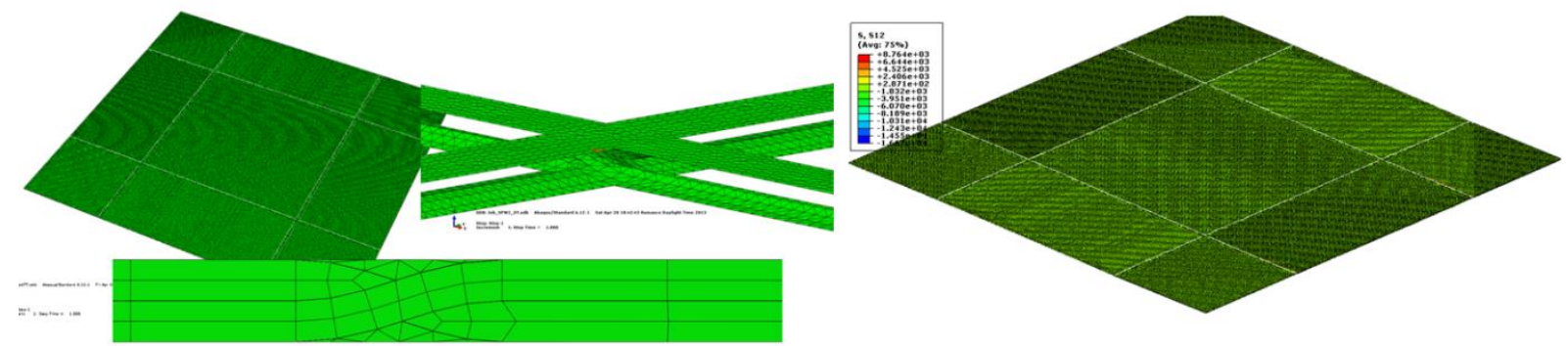

(b)

Figure 17 
$\underline{\text { Tables }}$

\begin{tabular}{|c|c|c|}
\hline \multicolumn{3}{|c|}{ Satin weave fabric properties } \\
\hline Carbon fibre type & \multicolumn{2}{|c|}{ T300J } \\
\hline Direction & warp & weft \\
\hline RUC width, $[\mathrm{mm}]$ & \multicolumn{2}{|c|}{7.4} \\
\hline Number of measurements & 20 & 20 \\
\hline Yarn width, $[\mathrm{mm}]$ & $1.31 \pm 0.01$ & $1.32 \pm 0.08$ \\
\hline Yarn thickness, $[\mathrm{mm}]$ & $0.162 \pm 0.01$ & $0.161 \pm 0.06$ \\
\hline Yarn spacing, [mm] & $1.46 \pm 0.04$ & $1.48 \pm 0.07$ \\
\hline Linear mass density, $[$ Tex] & \multicolumn{2}{|c|}{198} \\
\hline Yarn filament count & \multicolumn{2}{|c|}{3000} \\
\hline Filament diameter, $[\mathrm{mm}]$ & \multicolumn{2}{|c|}{0.007} \\
\hline Carbon fibre density, $\left[\mathrm{g} / \mathrm{cm}^{3}\right]$ & \multicolumn{2}{|c|}{1.75} \\
\hline \multicolumn{3}{|c|}{ Mechanical properties of the PPS matrix } \\
\hline Melting temperature, $\left[{ }^{\circ} \mathrm{C}\right]$ & \multicolumn{2}{|c|}{280} \\
\hline Glass transition temperature, $\left[{ }^{\circ} \mathrm{C}\right]$ & \multicolumn{2}{|c|}{90} \\
\hline Optimal crystalline temperature, $\left[{ }^{\circ} \mathrm{C}\right]$ & \multicolumn{2}{|c|}{$150-160$} \\
\hline Tensile strength, $[\mathrm{MPa}]$ & \multicolumn{2}{|c|}{90} \\
\hline Shear strength, [MPa] & \multicolumn{2}{|c|}{63} \\
\hline Ultimate strain & \multicolumn{2}{|c|}{0.08} \\
\hline Tensile Young's modulus, [MPa] & \multicolumn{2}{|c|}{3800} \\
\hline
\end{tabular}

Table 1

\begin{tabular}{|c|c|c|}
\hline$x= \pm \frac{|A B|}{2}$ & $y= \pm \frac{|A C|}{2}$ & $z= \pm \frac{|A D|}{2}$ \\
\hline$u\left(\frac{|A B|}{2}, y, z\right)=u\left(-\frac{|A B|}{2}, y, z\right)+u_{A}-u_{B}$ & $u\left(x, \frac{|A C|}{2}, z\right)=u\left(x,-\frac{|A C|}{2}, z\right)+u_{A}-u_{C}$ & $u\left(x, y, \frac{|A D|}{2}\right)=u\left(x, y,-\frac{|A D|}{2}\right)+u_{A}-u_{D}$ \\
\hline$v\left(\frac{|A B|}{2}, y, z\right)=v\left(-\frac{|A B|}{2}, y, z\right)+v_{A}-v_{B}$ & $v\left(x, \frac{|A C|}{2}, z\right)=v\left(x,-\frac{|A C|}{2}, z\right)+v_{A}-v_{C}$ & $v\left(x, y, \frac{|A D|}{2}\right)=v\left(x, y,-\frac{|A D|}{2}\right)+v_{A}-v_{D}$ \\
\hline$w\left(\frac{|A B| a}{2}, y, z\right)=w\left(-\frac{|A B|}{2}, y, z\right)+w_{A}-w_{B}$ & $w\left(x, \frac{|A C|}{2}, z\right)=w\left(x,-\frac{|A C|}{2}, z\right)+w_{A}-w_{C}$ & $w\left(x, y, \frac{|A D|}{2}\right)=w\left(x, y,-\frac{|A D|}{2}\right)+w_{A}-w_{D}$ \\
\hline
\end{tabular}

Table 2

\begin{tabular}{|c|c|c|c|c|c|}
\cline { 2 - 6 } \multicolumn{1}{c|}{} & WiseTex - MeshTex & A-M1 & B-M2 & B-M3 & B-M4 \\
\hline Fibre volume fraction (Yarn), [\%] & 70 & 70 & 70 & 70 & 70 \\
\hline Volume Matrix (RUC), $\left[\mathrm{mm}^{3}\right]$ & 5.684 & 5.602 & 5.526 & 5.526 & 5.481 \\
\hline Volume Yarns (RUC), $\left[\mathrm{mm}^{3}\right]$ & 11.784 & 11.733 & 11.809 & 11.984 & 11.984 \\
\hline Volume Total (RUC), $\left[\mathrm{mm}^{3}\right]$ & 17.468 & 17.335 & 17.335 & 17.510 & 17.465 \\
\hline Fibre volume fraction (RUC), [\%] & 47.223 & 47.380 & 47.687 & 47.791 & 48.03 \\
\hline
\end{tabular}

Table 3 


\begin{tabular}{|c|c|c|c|c|c|c|c|c|c|}
\cline { 2 - 11 } \multicolumn{1}{c|}{} & PBC ORAS & PBC SotA & A-M1-G2 & B-M2-G2 & B-M3-G1 & B-M3-G2 & B-M3-G3 & B-M4-G2 & Experiment \\
\hline $\mathrm{E}_{11},[\mathrm{GPa}]$ & 56.56 & 56.50 & 55.86 & 56.22 & 56.24 & 56.20 & 57.33 & 56.20 & $57 \pm 1$ \\
\hline $\mathrm{E}_{22},[\mathrm{GPa}]$ & 56.54 & 56.47 & 55.84 & 56.20 & 56.20 & 56.20 & 57.12 & 56.21 & $57 \pm 1$ \\
\hline $\mathrm{E}_{33},[\mathrm{GPa}]$ & 10.55 & 10.53 & 10.68 & 10.75 & 10.66 & 10.66 & 10.63 & 10.66 & - NA- \\
\hline $\mathrm{v}_{12},[-]$ & 0.08 & 0.08 & 0.08 & 0.08 & 0.08 & 0.08 & 0.07 & 0.08 & $0.05 \pm 0.02$ \\
\hline $\mathrm{v}_{13},[-]$ & 0.42 & 0.42 & 0.42 & 0.42 & 0.42 & 0.42 & 0.42 & 0.42 & $-\mathrm{NA}-$ \\
\hline $\mathrm{v}_{23},[-]$ & 0.42 & 0.42 & 0.42 & 0.42 & 0.42 & 0.42 & 0.42 & 0.42 & - -NA- \\
\hline $\mathrm{G}_{12},[\mathrm{MPa}]$ & 4354.48 & 4344.91 & 4382.51 & 4404.63 & 4399.86 & 4398.32 & 4439.57 & 4390.28 & $4360 \pm 60$ \\
\hline $\mathrm{G}_{13},[\mathrm{MPa}]$ & 3179.14 & 3160.52 & 3214.53 & 3244.98 & 3231.36 & 3228.67 & 3246.34 & 3227.19 & $-\mathrm{NA}-$ \\
\hline $\mathrm{G}_{23},[\mathrm{MPa}]$ & 3180.31 & 3160.85 & 3212.70 & 3245.06 & 3231.07 & 3228.96 & 3239.40 & 3228.68 & $-\mathrm{NA}-$ \\
\hline
\end{tabular}

Table 4 